\title{
Designing neural networks for the prediction of the drilling parameters for Kuwait oil and gas fields
}

\author{
Abdulrahman F. Al-Rashidi \\ West Virginia University
}

Follow this and additional works at: https://researchrepository.wvu.edu/etd

\section{Recommended Citation}

Al-Rashidi, Abdulrahman F., "Designing neural networks for the prediction of the drilling parameters for Kuwait oil and gas fields" (2000). Graduate Theses, Dissertations, and Problem Reports. 1101. https://researchrepository.wvu.edu/etd/1101

This Thesis is protected by copyright and/or related rights. It has been brought to you by the The Research Repository @ WVU with permission from the rights-holder(s). You are free to use this Thesis in any way that is permitted by the copyright and related rights legislation that applies to your use. For other uses you must obtain permission from the rights-holder(s) directly, unless additional rights are indicated by a Creative Commons license in the record and/ or on the work itself. This Thesis has been accepted for inclusion in WVU Graduate Theses, Dissertations, and Problem Reports collection by an authorized administrator of The Research Repository @ WVU. For more information, please contact researchrepository@mail.wvu.edu. 


\title{
Designing Neural Networks for the Prediction of the Drilling Parameters for Kuwait Oil and Gas Fields.
}

\author{
By \\ Abdulrahman F. AL-Rashidi \\ A thesis submitted to the College of Engineering and Mineral Resources \\ At West Virginia University
}

In partial fulfillment of the requirements for the degree of Master of Science

In

Petroleum and Natural Gas Engineering

H. I. Bilgesu, Ph.D., Chair.

S. Ameri.

K. Aminian, Ph.D.

D. Della-Giustina, Ph.D.

Department of Petroleum and Natural Gas Engineering

West Virginia University

Morgantown, West Virginia

1999

Keywords: Neural Networks, Drilling, Optimization, Kuwait, Drilling Parameters, Bit planning. 


\begin{abstract}
Designing Neural Networks for the Prediction of the Drilling Parameters for Kuwait Oil and Gas Fields.
\end{abstract}

\title{
Abdulrahman F. Al-Rashidi
}

In this study a new methodology was developed to predict the drilling parameters using the Artificial Neural Network. Three models were developed to predict bit type, rate of penetration (ROP), and cost-per-foot (cost/ft), respectively.

The prediction of bit type and other drilling parameters from the current available data is an important criterion in selecting the most cost efficient bit. History of bit runs plays an important factor in bit selection and bit design. Based on field data, the selection of bit type can be accomplished by the use of a neural network as an alternative bit selection method.

Three drilling parameters were modeled with data from different fields located in Kuwait. Results show that the drilling parameters of the new well can be predicted with the neural network models developed from the previous wells, a cost efficient alternative. 
This Thesis and every single thing I own, have, and achieved is dedicated to my inspirational mentor ra3i ash3ala "Falah bin Basman" my father, and algabla w alghalya "Munira" my mother. 


\section{ACKNOWLEDGEMENTS}

First and for most, thanks to Allah without Whose blessings none of this would be possible today. I would like to express my candid thanks and appreciation to all of the committee members. Special thanks go to my advisor and committee chairman Dr. Ilkin Bilgesu for his outstanding support toward the completion of this thesis. Thanks to Professor Sam Ameri, the chairman of the department, for his help and cooperation throughout my entire academic life at West Virginia University. I am also grateful to Dr. Kashy Aminian for his sincere contribution and help towards the completion of my MS. Special thanks are due to Dr. Della-Giustina to be one of the committee members.

The Author is grateful to his company "Kuwait Oil Company", specially the drilling department and Rig five. Special thanks are due to Baker Hughes, Kuwait division, for supplying the author with the needed information for this thesis.

I would like to acknowledge my brothers and sisters, family, onny "ammy adan amod" friends. I'd like to tell them that they were the best, and my sole calamity in times of distress and hardship. To all of you, I say " thanks and without all of you, this wouldn't have been achieved". 


\section{TABLE OF CONTENTS}

\section{Page}

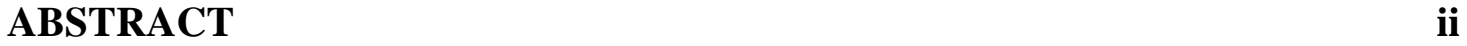

ACKNOWLEDGEMENTS iv

TABLE OF CONTENTS V v

LIST OF FIGURES vii

LIST OF TABLES $\quad$ x

1 INTRODUCTION 1

2 OBJECTIVE 4

3 PREVIOUS WORK $\quad 5$

4 LITERATURE 9

$\begin{array}{lll}\text { 4.1 ROLLER CONE BITS } & 10\end{array}$

$\begin{array}{lll}\text { 4.1.1 } & \text { Types } & 10\end{array}$

4.1.1.1 Steel Tooth Bits $\quad \mathbf{1 0}$

4.1.1.2 Tungsten Carbide Insert Bits $\quad 11$

4.1.2 $\quad$ Factors that affect the Roller Bits performance 12

$\begin{array}{llr}\text { 4.1.2.1 } & \text { Formation Factor }\end{array}$

4.1.2.2 Weight on Bit and Rotary-speed Factors 14

$\begin{array}{lll}\text { 4.1.2.3 Hydraulics Effects } & 14\end{array}$

$\begin{array}{lll}\text { 4.1.3 } & \text { Classification } & 16\end{array}$

$\begin{array}{lll}\text { 4.2 DRAG BITS. } & 17\end{array}$

$\begin{array}{lll}\text { 4.2.1 } & \text { Types } & 17\end{array}$

$\begin{array}{lll}\text { 4.2.1.1 Diamond Bits } & 17\end{array}$

4.2.1.2 Polycrystalline Diamond Compact Bits (PDC) 17

4.2.1.3 Drag Bits with Blades Cutter Elements 18 
4.2.2 Factors Effecting Diamond Bits Performance 19

4.2.2.1 $\quad$ Formation Factor 19

4.2.2.2 Weight on Bit, Rotary Speed, and Circulation Rate Factors 19

4.2.2.3 Hydraulics Factors 19

5 APPROACH 21

$\begin{array}{llr}5.1 \text { Artificial Neural Networks } & 21\end{array}$

$\begin{array}{lll}5.2 & \text { Data Acquisition } & 23\end{array}$

$\begin{array}{llr}5.3 \text { Procedure } & 26\end{array}$

5.3.1 Data Preparation 26

5.3.1.1 Input Data Selection $\quad 26$

5.3.1.2 Non-numeric Data Conversion 26

5.3.2 Network Preparation 29

5.3.2.1 Preparation 29

$\begin{array}{lll}\text { 5.3.2.2 } & \text { Training } & 31\end{array}$

6 RESULTS AND DISCUSSION 34

6.1 Kuwait-1 data set 36

6.2 Kuwait-2 data set 39

6.3 Kuwait-3 data set 41

6.4 Kuwait-4 data set 43

6.5 Kuwait-5 data set 45

$\begin{array}{ll}\text { 6.6 Summary } & 47\end{array}$

7 CONCLUSIONS

REFERENCES $\quad 54$

APPENDIX [A]

$\begin{array}{lr}\text { APPENDIX [B] } & 61\end{array}$ 


\section{LIST OF FIGURES}

\section{$\underline{\text { Figure }}$}

1

2

3

4

5

6

7

8

9

10

11 Comparison of neural network predicted cost per foot with the measured cost per foot. Kuwait-3 NN3 Run (3.C).

12 Comparison of neural network predicted bit type with the selected bit type. For (V2 code) Kuwait-3 NN1 Run (4).

13 Comparison of neural network predicted bit type with the selected bit type. For (bit code) Kuwait-3 NN1 Run (4.A).

14 Comparison of neural network predicted ROP with the measured ROP. Kuwait-4 NN2 Run (4.B). $\underline{\text { Page }}$

60

61

61

62

64

64

65

65

66

66

67 
16 Comparison of neural network predicted bit type with the selected bit type. For (bit code) Kuwait-5 NN1 Run (5.A).

17 Comparison of neural network predicted ROP with the measured ROP. Kuwait-5 NN2 Run (5.B).

Comparison of neural network predicted cost per foot with the measured cost per foot. Kuwait-5 NN3 Run (5.C).

Contribution of input parameters for the bit type prediction. Kuwait-1 NN1 Run (1.A).

Contribution of input parameters for the ROP prediction. Kuwait-1 NN2 Run (1.B).

Contribution of input parameters for the cost per foot prediction. Kuwait-1 NN3 Run (1.C).

Contribution of input parameters for the bit type prediction. Kuwait-2 NN1 Run (2.A).

23 Contribution of input parameters for the ROP prediction. Kuwait-2 NN2 Run (2.B).

Contribution of input parameters for the cost per foot prediction.

Kuwait-2 NN3 Run (2.C).

Contribution of input parameters for the bit type prediction. Kuwait-3 NN1 Run (3.A).

Contribution of input parameters for the ROP prediction. Kuwait-3 NN2 Run (3.B).

Contribution of input parameters for the cost per foot prediction. Kuwait-3 NN3 Run (3.C).

Contribution of input parameters for the bit type prediction. Using (V2 code), Kuwait-4 NN1 Run (4).

Contribution of input parameters for the bit type prediction. Using (bit code), Kuwait-4 NN1 Run (4.A).

Contribution of input parameters for the ROP prediction. Kuwait-4 NN2 Run (4.B). 
31 Contribution of input parameters for the cost per foot prediction. Kuwait-4 NN3 Run (4.C).

32 Contribution of input parameters for the bit type prediction. Kuwait-5 NN1 Run (5.A).

33 Contribution of input parameters for the ROP prediction. Kuwait-5 NN2 Run (5.B).

34 Contribution of input parameters for the cost per foot prediction. Kuwait-5 NN3 Run (5.C). 


\section{LIST OF TABLES}

Table

1.

2.

3.

4.

5.

6.

7.

8.

9.

10. $\underline{\text { Description }}$

Page

List of runs with input and output parameters

The range of values for Kuwait-1 data set.

36

The range of values for Kuwait-2 data set.

The range of values for Kuwait-3 data set.

The range of values for Kuwait-4 data set.

The range of values for Kuwait-5 data set.

Comparison of results for bit type predictions.

Comparison of results for ROP predictions.

Comparison of results for cost per foot predictions.

Kuwait Energy Overview 


\section{INTRODUCTION}

Optimization of the drilling parameters and bit selection planning are considered as unique challenges to drilling engineers. Correct drilling parameters have becoming increasingly important given the high cost of drilling a well. Numerous efforts have been made in an effort to predict the bit capable of achieving the highest rate of penetration. Bit selection programs that give the highest rate of penetration have been prepared by engineers and technicians from rock bit manufactures as a service to oil companies to reduce the drilling cost.

Achieving the optimal rate of penetration with the least possible bit wear is the aim of every drilling engineer when selecting a drilling bit. There are several factors that can effect the rate of penetration, which are ranked as follows:

1. Formation properties.

2. Bit type.

3. Bit weight.

4. Bit rotational speed.

5. Bit hydraulics.

Obviously, formation properties can not be changed before drilling and thus selection of the correct bit type plays a major importance in achieving high rates of penetration. Unfortunately, there are no foolproof methods of selecting the optimum drilling bit for a formation to be drilled. Bit selection, like the selection of the correct weight on bit (WOB), rotation-per-minute (RPM), and hydraulics, is dependent upon a degree of trial and error. The aim of any bit selection tool is to reduce the trial and error factor to a minimum. There are 
many proposed methods for bit selection and often more than one is used before reaching a decision. Bit selection methods include:

1. Cost analysis. The cost per interval drilled is the most commonly used method for comparing the performance of various bits. The basic equation used is as follows:

Cost per foot,$(\$ / f t)=\frac{\text { Bit Cost }, \$+(\text { Rotating time }, h r+\text { Trip time }, h r) * \text { Hourly Rig } \cos t, \$ / h r}{\text { Footage }, f t}$

2. Dull bit evaluation.

3. Offset well bit record analysis.

4. Offset well logs analysis.

5. International Association of Drilling Contractor (IADC) Bit Coding.

6. Manufacturer's product guides.

7. Geophysical data analysis.

8. General geological consideration.

Differences in formation and drilling techniques require a large variety of bit types to minimize costs. Because the efficiency of any drilling operation depends largely upon the right choice of the most suitable bit for a given set of conditions, there is a need for further research on methods and guidelines that could simplify the task. Thus, the artificial neural network was used as the new methodology to predict the drilling parameters of bit type, rate of penetration, and cost per foot for drilling optimization

Artificial Neural Network (ANN), also referred to as Neural Network (NN), is a data processing system consisting of a large number of interconnected processing elements configured in a manner that was inspired by the structure of the cerebral cortex portion of the brain. Neural network offers a new form of information processing that is fundamentally 
different from a traditional processing system. The system uses a knowledge base of various drilling parameters, to produce a "correlation" description of the most suitable bit, ROP, and cost-per-foot for a particular set of drilling parameters.

The "correlation" is derived from various drilling parameters, which may include some or all of the following data from a field or a region:

bit type, bit size, total flow area, depth in, depth out, drilled interval, drilling hours, ROP, minimum WOB, maximum WOB, minimum RPM, maximum RPM, mud flow rate, mud weight, circulating pressure, the bit inner row dull grading, the bit outer row dull grading, cost of bit in dollars (Cost \$), cost-per-foot, and well name.

Compared to conventional bit selection methods, this approach makes a more effective use of past experience leading to a higher and more uniform level of bit selection expertise in drilling operations. 


\section{OBJECTIVE}

The objective of this study was to test the hypotheses of three drilling models using data gathered from the field and then develop a practical method that can be applied in the field for bit selection, ROP, and cost-per-foot to minimize drilling costs. This study will be implemented by using Artificial Neural Networks as a new methodology to predict these parameters.

Data on most recent wells drilled in Kuwait were used in this study to analyze and compare the effectiveness of different bit types and drilling parameters for optimum penetration of the various formation and depth intervals common to Kuwait and the Middle East area. This study was initiated in an attempt to improve penetration rates and reduce per well drilling cost. Based on the research done for this study, an optimum bit selection, ROP, and cost per foot can now be incorporated into drilling plans for subsequent wells to be drilled in the area. 


\section{PREVIOUS WORK}

Achieving the highest rate of penetration with the least possible bit wear is the aim of every drilling engineer when selecting a drilling bit. Correct bit selection has become increasingly important given the rises in expenses involved in drilling a well. This has meant that oil companies engage in a perpetual struggle to predict the ideal bits for optimum rates of penetration. Due to the demands of oil companies for top-notch bit selection programs, engineers and technicians are hired away from rock bit manufactures for their expertise in processes that reduce drilling costs. The current criteria for bit selections are based upon the cost-per-foot, and the analysis of offset wells bit records.

\section{Cost per foot analysis}

The cost per interval drilled is the most commonly used method for comparing the performance of various bits. The basic equation used is as follows:

$$
\text { Cost per foot },(\$ / f t)=\frac{\text { Bit Cost }, \$+(\text { Rotating time, } h r+\text { Trip time, } h r) * \text { Hourly Rig } \cos t, \$ / h r}{\text { Footage, } f t}
$$

However, the cost per foot is not an ideal measure for bit selection. Cost per foot Equation shows that cost per foot is controlled by five variables. For a given bit cost (B) and hole section (F), cost-per-foot will be highly sensitive to changes in rig cost-per-hour (R), trip time $(\mathrm{T})$, and rotating time (t). The trip time may not be always easy to determine, unless a straight running in and pulling out of the hole is made. If the bit is pulled out for some other reason, such as to case a shoe for a wiper trip, care must be taken not to add this time to either the trip time or the rotating time. Rig cost-per-hour will greatly influence the value of cost-per- 
foot. For a given hole section and penetration rate in a field drilled by various rigs, having a variety of cost-per-hour, the same bit will produce different values of cost-per-foot.

The two major sources of uncertainty in this decision are pointed out as:

1. Inaccuracies in the measurement and prediction of footage, trip time, and rotating time..

2. Lack of precise knowledge of formation changes affecting present and future penetration rates.

\section{Bit record analysis}

This is based upon a comparison of offset well data, which is usually stored in a database program. Database tracks records of data history, and then a bit program can be selected based on best performance achieved for the next well to be drilled. Preparation of a reliable bit program requires the collection of data from every possible source. Several authors have suggested that the following data be collected before a well program can be initiated:

1. Specifications for the proposed well should include the hole sizes, casing programs, mud programs, and anticipated hole programs.

2. Offset well data should include bit records, mud reports, electric logs, mud logs, and drilling curves.

3. Effects of altering drilling variables such as mud hydraulics, drilling parameters, and bit types.

4. Seismic data given the estimated geological formation tops and interval thicknesses, well location, drilling contractor, spud date, etc.

5. Geological information, formation description, and formation type (hard, soft, abrasive, etc). 
The bit record not only indicates the bit types but also the drilling parameters used, penetration rate, effect of drilling fluids on rate of penetration, and the effect of changes in hydraulic conditions on the drilling rate. It becomes apparent from the bit record that the drilling fluid properties along with the hydraulic operation parameters have a direct effect on overall bit performance. Electric logs can be useful for bit selection because they are capable of determining formation tops and geological base on sand and shale content of the formations. Bit selection is affected significantly in areas where electric log interpretation is most critical

For example, in Kuwait bit optimization is dependent on offset-well bit data. A bit database was developed into which all offset-well bit data were loaded. Bit records for a single well as well as the best performing bits in a field can be extracted from the database. The bit database was used to set benchmarks for different fields and compare bit performance when evaluating trial runs. (Alsaleh, 1999)

\section{$\underline{\text { Artificial Neural Network }}$}

In recent years there have been many studies describing models and methods to predict and interpret the drilling parameters to optimize drilling operation and reduce drilling costs. Relatively few of these models have proven to be practical in the field, or have been able to establish validity over a wide number of environments. One of the new methods to predict the drilling parameters is using an artificial neural network.

Artificial neural networks have been used since the 1980s. They are very helpful in solving petroleum engineering problems. Examples of petroleum engineering projects that benefit from the help of neural networks include reservoir characterization, zone identification, well testing, and drilling optimization. (Altmis, 1996) 
Altmis (1996) has used neural networks to predict the drilling parameters. She used a set of data generated by an advanced, full size drilling rig simulator. The parameters used to train the neural network were RPM, time, bit type, WOB, rotary torque, ROP, formation abrasiveness, formation drillability, bit bearing wear, tooth wear, and SPM. Some of Altmis's data was obtained from fields in the United State, but only RPM, time, bit type, WOB, rotary torque, ROP, and SPM parameters were included for prediction. It is important to note that Altmis used only three bits in her study.

In another area of petroleum, White, et.al (1998) used several artificial neural networks to design and develop zone identification in a complex reservoir. In this study, several ANNs were successfully designed and developed for zone identification in heterogeneous formations from geological well logs. Reservoir characterization plays a critical role in appraising the economic success of reservoir management and development methods. Nearly all reservoirs show some degree of heterogeneity, which invariable impact the production. As a result, the production performance of a complex reservoir can not be realistically predicted without accurate reservoir description. The difficulty stems from the fact that sufficient data to accurately predict the distribution of the formation attributes are not usually available. One of the key issues in the description and characterization of heterogeneous formations is the distribution of various zones and their properties. 


\section{LITERATURE}

The choice of bit to use depends on several factors. One is the type of formation to be drilled. The driller must determine whether the formation is hard, soft, medium-hard, or medium soft. Another item is the cost of the bit. Achieving the most wear possible from a bit cuts down bit costs and minimizes the number of trips needed for bit changes, although continuing to use a bit that is drilling very slowly is a false economy.

Formations vary significantly in hardness and abrasiveness, and will impact the performance of the bit. If there were no differences in rock formations, the driller could select only one bit, set the bit weight, rotary speed, and pump pressure, and then drill ahead at the maximum rate. Sometimes such a situation does exist, but usually the formations consist of alternating layers of soft material, hard rocks, and abrasive sections. Changing the bit every time there is a change in the formation is not always practical. Instead, one must choose a bit that represents a compromise, one that performs reasonably well under all conditions it must meet. Choosing such a bit is easier for a well to be drilled in a field where formations are known than for a wildcat where the formations are being met for the first time. The two types of bits used in rotary drilling are roller cone, and drag bits. 


\subsection{ROLLER CONE BITS}

The designer of roller cone bits, also known as rock bits, need to use heavy-duty bearings, a high-strength cone shell, and full-length cutting teeth. Each feature competes with the others for the limited space available to build the cutting structure on the roller cones. In addition, the designer must balance the toughness of steel against the brittleness of hardsurfacing materials in determining the matter of long life and effective cutting. As a result, bit designers have developed several types of bits, each of which emphasizes a particular quality needed to drill a particular type of formation. (Leecraft, J., 1990)

\subsubsection{Types}

Rock bits may be classified in general as (1) Steel tooth (Milled tooth) bits and (2) Tungsten carbide insert bits.

\subsubsection{Steel Tooth Bits}

Steel tooth bits are formed by milling directly on the cone shell as it is manufactured. Steel-tooth bit can be designed for soft, medium, and hard formations. Cones offset and teeth sizes have their impact in bit design. For example, bits designed for the softest formations with the least amount of abrasive characteristics have the most cones offset and widely spaced, long, and sharp teeth. Where bits designed for hard formation have the least cones offset (or none) and more closely spaced, shorter, and stronger teeth. 


\subsubsection{Tungsten Carbide Insert Bits}

Tungsten carbide insert bits that are inserted into pre-drilled holes in the steel cone shell. Carbide insert bits were used to reduce trip time, because the same bit could be used on different formations; however, slow bit speeds reduce the rate of penetration and faster speeds could cause insert breakage.

Similar to steel tooth, tungsten carbide insert bits are available for soft, medium, and hard formation. For the hardest formations, a hemispherical-shaped end is used on the inserts, chisel-shaped inserts for medium formations, and larger-diameter, sharper crested, and more widely spaced inserts for very soft formations. New developments in cone materials have made the cones more wear resistant, cutting down on cone failure. The most prominent innovation in carbide insert bits has been the development of sealed bearings, since bearing failure was one of the more common failures of this type of bit.

The advantages of the carbide insert bit include great drillability, good insert burial into the formation, up to $80 \%$ of the insert-per-revolution in soft formations, and the ability to drill different types of formations with the same bit. Their disadvantages include the erosion around the base of inserts that can result in their loss, and the possibility that (with complete insert burial) an area of the cone shell can come into contact with the formation and transmit shock loads from the drill string directly to the bearing. 


\subsubsection{Factors that affect the Roller Bits performance}

The formation, bit weight, rotary speed, and bottom-hole cleaning velocity factors all affect the performance of a roller cone bit. Another factor that effects the bit performance is the experience of the driller. The parameters that the driller applies to the bit have their great impact on the bit. The driller can improve the bit performance by paying attention to the variables of formation, bit design, and rig operating practices

\subsubsection{Formation Factor}

Most rock bits will make fair progress in the majority of the formations it encounters. However, to obtain maximum footage and penetration rates and thus lower drilling costs, a type of rock bit designed for the specific formation being drilled should be selected. Roller bits are available for soft, medium-soft, medium-hard, and hard formations. International Association of Drilling Contractor (IADC) has developed a standard classification code that is used to classify bits made by different manufactures according to the hardness of the rock that they are designed to drill. IADC code includes a description of the practical design feature of the bit.

Steel tooth bit may be used on soft formations if the bit has deep and widely spaced teeth with sufficient scraping action on bottom. The scraping is obtained by the offset of the roller cones. In soft formation, the teeth should be widely spaced to prevent the bit from balling up, which occurs when the formation material packs so tightly between the teeth that the mud stream can not keep them clean.

For medium-soft formations offsets of lesser degrees can be employed to give a twisting-scraping action to the teeth on the bottom of the hole. Since depth of penetration is not as great as that in softer rocks, slightly shorter teeth are used. Also an increase in the number and strength of the teeth is needed for the bit to have a longer life. 
Medium-hard formations such as hard limestone, dolomite, and hard shale are too hard and perhaps too abrasive. Therefore, bit cones for this type of formation are given a slight offset, and teeth are closely spaced. For formations that are abrasive but of relatively low strength, hard-facing material is applied to teeth and gauge surfaces because they may be subjected to more severe wear than those used for soft formations. Where for formations that have high compressive strength and require heavy weight for effective crushing and chipping, maximum performance can be obtained without hard facing material.

Similar to steel tooth bit, tungsten carbide insert bits can also be used to drill hard, medium-hard, medium, and soft formations. For the hardest formations, a hemispherical shaped end is used on the inserts. The inserts are closely spaced, thus the exposed hemispherical shaped ends of the inserts produce a chipping and crushing action on the rock, thereby drilling hole as the bit is rotated under load.

For medium-hard formations the bits have greater projection of inserts above the steel cones, deeper ventilation grooves, and conical-shaped ends on the inserts. They drill faster in medium-hard formations than bits with inserts that have rounded ends. The use of chisel-shaped inserts for medium formations has led to the successful application of this type of bit for drilling shale and other softer plastic formations. As with steel tooth bits, it is necessary to produce a design for scraping and gouging action, an offset design, rather than to rely on the crushing type of action used by the hard-formation type of bits. Tungsten carbide inserts have been successfully applied in the very soft formations by using larger-diameter, sharper-crested, more widely spaced inserts, maximum scraping and gouging action, a thicker and more abrasiveresistant carbonized case on the cone metal for retaining the inserts, and long-life journal bearings. (Leecraft, J., 1990) 


\subsubsection{Weight on Bit and Rotary Speed Factors}

Field experience with steel-tooth bits has proven that drilling rate in brittle rocks increase more than proportionally to increases in drilling weights. Sometimes increasing the weight on bit would increase the rate of penetration. This is true for short-term improvement in drilling rates, but it is not for overall performance.

Achieving maximum rock bit performance depends on tooth form and structure as well as on bearing life. Soft formations restrict the use of heavy weight because of the tendency of the steel-tooth bit to ball up. It is possible to increase rotary speed in order to offset the reduced weight, and good cleaning by high fluid velocity allows fast penetration rates in such formations. Precaution must be taken when increasing the fluid velocity, to prevent hole washout. In harder formations, rotary speed is limited by abrasive properties of the formation, where in this case it is necessary to use heavier weights to exceed the strength of the formation. Excessive rotary speeds with heavier weight in such formation would result in tooth and bearing wear. The experience of the driller is critical in this case, and the parameters applied to the bit will effect the overall bit performance.

The procedures for determining the best weight and rotary speed to use with steel tooth bits also apply to tungsten carbide insert bits. The principal difference is that under normal conditions the cutting structure (i.e., the teeth) of a tungsten carbide insert bit wears very little. Therefore, weight and rotary speed may remain constant.

\subsubsection{Hydraulics Effects}

Drill bit hydraulics is generally associated with the use of jet bits. The purpose of the jet nozzles is to improve the cleaning action of the drilling fluid at the bottom of the hole. The removing action of the cuttings plays an important factor in drilling operation. When cuttings 
are not removed from underneath the bit, several drilling problems will occur such as wasteful bit wear and drilling time and therefore higher drilling costs.

Watercourses were the first to be integrated into a roller cone bit and are still in limited use today. These courses direct drilling fluid onto the cutters to keep them clean, and the fluid then goes on to clean the hole. The velocity of the stream of drilling fluid in this type of passageway is relatively low, and the disadvantages of the system include balling and cone erosion. Such conventional watercourses have been almost completely replaced by jet watercourses, or nozzles. These nozzles direct the stream of drilling fluid past the cones and completely flush out cuttings in the hole. The stream of drilling fluid can be controlled by changing the nozzle size and can improve the rate of penetration in soft formations by washing away or eroding the formation even before the bit touches the bottom of the hole. (Leecraft, J., 1990)

Under steady state drilling conditions the rate of cuttings removal from under the bit must equal the rate at which new chips are formed. This implies that penetration rate can be controlled by the cutting generation process, removal process, or a combination of the two processes.

Parameters such as jet impact force, hydraulic horsepower, jet velocity, and jet Reynolds Number have been used in attempts to quantify the effect of bit hydraulics on penetration rate. All of these parameters refer to properties of the fluid at the time it exits the jet nozzle. The two parameters most commonly used to quantify the effect of hydraulics on penetration rate are jet impact force and hydraulic horsepower per square inch of bit area (HSI). Maximum horsepower is obtained with slightly smaller nozzles than are required for maximum impact force. 
The effect of hydraulics on penetration rate is greatest at high weight-on-bit and is greater for soft rocks than for harder rocks. The increased ROP due to increased hydraulics tends to reach a plateau and level out at some point depending on the bit type, rpm, WOB, and rock strength. A given change in hydraulics will affect the penetration rate of short tooth bits more than long tooth bits if all else is constant. (Azar, J., 1995)

\subsubsection{Classification}

A standard system to classify roller cone bits has been developed by IADC. Bits are classified according to type (milled tooth or insert bits), kinds of formation for which they are

designed (expressed by series and type), mechanical features, and manufacturer. The system of classification permits comparison of the bit types offered by various bit manufacturers. (Gabolde, G., J., Nguyen., 1999) 


\subsection{DRAG BITS.}

Unlike rolling cutter bits, drag bits do not have any rolling parts that require strong and clean bearing surface. There are three general types of drag bits that are in common usage, which are Drag Bits with blade (steel) cutter elements, diamond bits, and polycrystalline diamond compact (PDC).

\subsubsection{Types}

\subsubsection{Diamond Bits}

Diamond bit is more expensive than roller bits, it may cost three or four times as much as a carbide insert bit, and several times as much as a steel-tooth bit. Sometimes diamond bit can offer an economic advantage over roller bit. The most important factor in its advantage is the fact that it makes more hole than any other bit over the entire period of its life. Diamond bits have two valuable characteristics, a basic design and no parts that move.

Diamond bits, in which industrial grade diamonds are set into bit heads that is manufactured by a powdered metallurgy technique. The size, shape, quantity, quality, and exposure of the diamonds are tailored to provide the best performance for a particular formation. Each bit is designed and manufactured for a particular job rather than being mass produced as roller cone bits are. The cuttings are removed by mud that flows through a series of water courses. The design of these water courses is aimed at forcing fluid around each individual diamond. The matrix diamond bit cuts rock by grinding and thus a primary function of the fluid is to conduct heat away from the diamonds.

\subsubsection{Polycrystalline Diamond Compact Bits (PDC)}

The PDC bits are made up of a layer of synthetic polycrystalline diamond that is bounded to a cemented tungsten carbide substrate in a high pressure, high temperature process. 
The cutters are generally much larger than natural diamonds and are designed to cut the rock by shearing, similar to metal machining (conventional drag bits). PDC bits have proven to be very successful in homogeneous and soft to moderate strength formations. In formations where they are successful, they can drill two to three times faster than a roller cone bit and may have an equally long life.

The diamond on PDC cutter is many times harder than the hardest rock that is drilled in normal oil and gas well drilling. When the bits were first being developed, it seemed almost impossible that the cutters could sometimes be worn out in only a few feet of drilling. This accelerated wear is often attributed to the cutters becoming overly hot. The PDC material generally contains small amounts of various metals located in the voids between diamond grains. Because of the differential thermal expansion of the diamond grains and binder metals, heating the cutters will cause high stresses that makes individual diamond crystals easier to break away from the cutter. Additionally, at higher temperature the diamond partially converts back into graphite, especially in an oxidizing atmosphere.

\subsubsection{Drag Bits with Blades Cutter Elements}

Although the grandfather of all rotary drag bits, "the fishtail," has been relegated to the museum of petroleum antiquities, not all drag bits have met the same fate. There are a few areas left where drag bits are still necessary.

The original type of fishtail bit had two blades and an eye for the drilling mud near the threaded shank of the bit. This bit ruled the oil fields from the early time until the 1920's. A modified fishtail drag bit that has actual jet bit was produced in 1947, with three or four blades of hard metal welded to the body of the bit. Bits of this type are occasionally used today for drilling soft, shallow formations prior to setting surface casing. They are commonly available 
in sizes up to 24 inches. Various sizes of bit bodies can be obtained for the complete range of blade sizes. Blades are expendable and designed to be completely worn out and thrown away.

\subsubsection{Factors Effecting Diamond Bits Performance}

\subsubsection{Formation Factor}

Diamond bits have three main cutting actions: compressive, abrasive, and plowing. Compressive action is the cutting action used most frequently. Diamonds in the bit create stresses in the formation that cause it to crack. The cracked pieces of formation are then pushed behind the diamond. Abrasive action accounts for about $15 \%$ of modern drilling applications. The bit drills this way when the formation is so hard and abrasive that the only way to get through it is to wear it away. Plowing action is a third type of action exercised by the diamond bit. When the bit can overcome the compressive strength of the rock so that the formation ruptures, the diamond can then scoop the formation in front of it.

\subsubsection{Weight on Bit, Rotary Speed, and Circulation Rate Factors}

Sufficient weight must be applied to cause the cutting points of the diamonds to penetrate the formation. The degree of penetration depends on the hardness and characteristics of the formation, the size and shape of the diamond point, and the applied unit weight. The weight causes penetration, and the rotation gives movement to the diamond, which removes the formation. The mechanical factors of weight and rotary speed are directly related to the drilling rate.

\subsubsection{Hydraulics Factors}

Hydraulics factors affect the drilling rate in relation to the efficiency with which these mechanical factors are applied. In regard to hydraulics, the jet principle, applied to the diamond bit, is the only efficient means of keeping the diamonds clean and cool so that new formations 
may be cut with each turn of the bit, thus increasing the effectiveness of the mechanical factors. The jet action, to be effective on a diamond bit, requires sufficient fluid velocity across the face of the diamond bit to clean and cool the diamond points satisfactorily. The ideal fluid velocity is always known, and usually other factors that may dictate a necessary change in this ideal velocity are also known. Therefore, for optimal performance, a diamond bit must not only be designed to meet hole conditions, but also have watercourses that give proper fluid distribution at the ideal velocity, based on the fluid available to the bit. The available fluid may be limited by the capacity of the mud pumps on the rig or by the fluid capacity of the diamond bit in use. (Leecraft, J., 1990) 


\section{APPROACH}

In this study a new methodology was developed to predict the drilling parameters using artificial neural networks. Three models were developed to predict the bit type, rate of penetration (ROP), and cost-per-foot, respectively.

The prediction of bit type and other drilling parameters from the current available data is an important criterion for reduction of drilling costs. History of bit runs is a very important factor in bit selection and bit design. The prediction of drilling parameters is complex because of the numerous variables that can affect the prediction. Mathematical models become more complex when too many variables are included, which lead to difficulties in evaluating drilling parameters. Based on field data, neural network applicability for bit type prediction was applied as an alternate method of bit selection.

\subsection{Artificial Neural Networks}

Unlike mathematical models that require precise knowledge of all parameters and their interrelation, neural networks can provide an estimation of the drilling parameters under various conditions without a precise knowledge of all contributing variables and their relationships. Neural network technology mimics the human brain's problem solving process. The networks, much like the brain, can apply knowledge gained from previous experiences to new problems. A neural network does so by building a system of neurons that are capable of making new decisions, classification, and forecasting.

The main interest in neural networks is rooted in the recognition that the human brain processes information in a different manner than conventional digital computers. Computers 
are extremely fast and precise at executing sequences of instructions that have been formulated for them. A human information processing is composed of neurons switching at speeds about a million times slower than a computer does. Yet, despite slower processing speeds, the human brain is more efficient than computers at such computationally complex tasks as speech and other forms of pattern recognition. Artificial neural networks, are physical cellular systems that can acquire, store, and use experiential knowledge. The knowledge is in the form of stable states or mapping embedded in networks that can be recalled in response to the presentation of cues.

Neural networks can help engineers and researchers by addressing some fundamental petroleum engineering problems as well as specific ones that conventional computing has been unable to solve. Petroleum engineers may benefit from neural networks on occasions when engineering data for design and interpretations are less than adequate.

Neural networks have shown great potential for generating accurate analysis and results from large amount of historical data that otherwise would not seem useful or relevant in the analysis. Neural networks have proved to be valuable pattern-recognition tools. They are capable of finding highly complex pattern within large amounts of data. 


\subsection{Data Acquisition}

There are several reasons for the apparent lack of commercial success. Many models have been derived using data from laboratory drilling tests. Since representative rock samples are hard to find, the experiments tend to cover a wide range of weight on bit and rotary speed, but in a narrow range of rocks. This tends to result in models, which attempt to simultaneously describe a wide range of effects (such as individual crater formation at low weights, through crater indexing, through to poor bit cleaning at the upper end of weight on bit). This leads to fairly complex models sometimes requiring four or more empirically derived parameters. Clearly this is impractical in field operations.

Laboratory data tends to be somewhat more ideal because data gathered in the field often contains a significant percentage of noise from the rather crude manner in which rig measurements of weight or rate of penetration are made. Therefore, laboratory data is often not practical because of the altered factors that can not be controlled in the field. Unlike laboratory experiments, the driller tends to drill a wider range of formations with a fairly narrow commercial range of weight on bit, rotary speed, and pump stroke, than would be seen in a laboratory experiment

The data for this study, recently gathered, was collected from different wells that drilled from different fields in Kuwait. A total of 13,234 set of data with 14 columns of variables was collected to feed the neural network. The gathered data consist of three different data; namely these data sets are Kuwait-1, Kuwait-2, and Kuwait-3.

Five different sets of data are used in this study where three are the main sets and two are subsets. The five sets are Kuwait-1, Kuwait-2, and Kuwait-3, Kuwait-4, and Kuwait-5. The 
main sets are Kuwait-1, Kuwait-2, and Kuwait-3. Where Kuwait-4 and Kuwait-5 are subsets created for further analysis for one specific region and one specific field, respectively. All of the three main sets were screened and improper data was eliminated prior to the study by the network. For example, Kuwait-1 consists of 3,526 set of data, only 1,893 set of data was used in this study. Similarly Kuwait-2, which consist of 6,700 set of data, only 4,466 set of data were used. Also Kuwait-3, which consist of 3008, only 2392 set of data were used. The data excluded were consisted of conditions such as reaming and coring operations, and drilled intervals less than 100 feet. Also precautions were taken to make the data identifiable by networks, because the networks are sensitive to certain types of data input and manners of identifying variables. The data sets, number of sets, and the drilling parameters for each set are listed as follow:

[A] Data set Kuwait-1 and Kuwait-2.

\begin{tabular}{|l|l||}
\hline DATA SET & NUMBER OF SETS \\
\hline Kuwait-1 & 1,893 \\
\hline Kuwait-2 & 4,466 \\
\hline
\end{tabular}

Both sets have the following drilling parameters:

Bit type, bit size, total flow area (tfa), depth in, depth out, drilled interval, drilling hours, ROP, minimum WOB, maximum WOB, minimum RPM, maximum RPM, flow rate, mud weight, circulating pressure, the bit inner row dull grading, the bit outer row dull grading, bit cost $(\$)$, cost per foot, and the well name. 
[B] Data set Kuwait 3, Kuwait 4, and Kuwait 5.

\begin{tabular}{||l|l||}
\hline DATA SET & NUMBER OF SETS \\
\hline Kuwait-3 & 2,392 \\
\hline Kuwait-4 & 450 \\
\hline Kuwait-5 & 367 \\
\hline
\end{tabular}

* Data set Kuwait-4 consisted of a combination of two different fields in one region where the formation tops are the same.

* Data set Kuwait-5 consisted of only one specific field.

All of the three sets (Kuwait-3, Kuwait-4, and Kuwait-5) have the following drilling parameters:

Bit type, bit size, total flow area, depth in, depth out, drilled interval, drilling hours, ROP, WOB, RPM, flow rate, circulating pressure, the bit inner row dull grading, the bit outer row dull grading, bit cost $(\$)$, cost per foot, and the well name. 


\subsection{Procedure}

\subsubsection{Data Preparation}

It is important to have reliable input data so that the output produced by the neural network can compute a good correlation. In optimizing the process, three major things have to be worked with to achieve a good $\mathrm{R}$ square value. In order for a good correlation, the input data must be prepared in advance. Two procedures were mainly involved in preparing the input data, which are identification of the input data, and conversion of non-numeric data to numeric.

\subsubsection{Input Data Selection}

Based on this study and every study using neural networks, the input variables play a critical role in achieving a good $\mathrm{R}$ square value. Identifying the most important inputs, which have great contributions and effects on the output is the main key to produce a good prediction. In this study most existing data were used and tested, in order to identify which variable to include.

\subsubsection{Non-numeric Data Conversion}

Neural networks accept only numeric input, a special way of coding the input data has to be designed. For example, if assigned 1 to Alaska, 2 to Alabama, and 50 to Washington, the system will read that Alaska is similar to Alabama, but Alaska is totally different than Washington, which is not true. In this study all the data were numeric except bit types and different approaches were used to convert non-numeric coding of bits. 


\section{Bit type coding}

Neural networks accept only numerical values, but the bit types from the gathered data were in letters. Due to the limitation of assigning a number to a variable, different ways of coding bit types were done prior to neural network application. For each different coding, several runs were done to compare and choose the best code that can represent the bit types. The different ways of coding the bit types, were as follow:

1. Coding bit types based on IADC bit coding, such as "111" for tooth bit with open bearing designed for soft formation, "415" for insert bit with sealed bearing and gauge protection designed for soft formation, and "M333" or "S316" for PDC bits where a number "1" was given to $\mathrm{M}$ and "2" to $\mathrm{S}$.

2. Coding bit types based on IADC bit coding, such as "111" for tooth bit with open bearing designed for soft formation, "415" for insert bit with sealed bearing and gauge protection designed for soft formation, and coding "M333" or "S316" PDC bits to its similar IADC code. PDC were coded based on Security DBS “PDC Roller bit comparison chart”.

3. Coding bit types based on IADC bit coding, such as "111" for tooth bit with open bearing designed for soft formation, "415" for insert bit with sealed bearing and gauge protection designed for soft formation, and excluding PDC bits.

4. Coding bit types based on IADC bit coding and include a manufacture company code, such as "111.1", where the first three codes describe the IADC code, and the last decimal digit describe the manufacture company. For example "111.1", "111.2", and "111.3" refer to three different companies. 
5. Coding bit types based on IADC bit coding and having an extra digit value "1111", "3415", "5333", and "7316" such that the first digit numbers are "1" for tooth, "3" for insert, "5" for M, and "7" for S. (V1).

6. Coding bit types same as code 5 , but divided each by 1000 , so that " 1111 " when divided by (1000.00) will equal “1.111”. (V2).

7. Coding bit type based on four digits used in step 5 with one code in one column. For example, the code "3415" will be as follows: "3" in column A, "4" in column B, "1" in column C, and "5" in column D.

8. Coding bit types based on one digit in one column similar to step 6 , but readjust "column B" to read same value for same formation type bits. For example both "115" tooth bit and "417" insert bit are designed for soft formations. Same digit, (number"1"), was assigned for both of them so that the network will identify them as having the same design in respond to formation type.

9. Assigning a number to each bit type starting with number " 1 " for a specific tooth bit designed for a soft formation (bit code). 


\subsubsection{Network Preparation}

The most important step in the process is to prepare the neural network for parameters to be studied. There are two major steps in using the network, namely preparation and training.

\subsubsection{Preparation}

The data file must be in a suitable format prior to selecting the inputs and the output. Most common formats are spreadsheet files or text files. The data is checked for proper input without missing parameters and the minimum and maximum values are determined. The maximum and minimum values determine the range of applicability of the neural network.

A portion of the data is set aside and the remaining are used in the training step. The data set aside is called test set and the most commonly used methods to select are by random, every Nth pattern, or after the Nth pattern. The amount of data set aside for testing generally constitutes 10 to $20 \%$ of the total available data. The remaining data is termed as the training set and used in the development of the neural network.

Several architectures are available in designing the neural network. In this study we have used standard "Backpropagation" and "Ward" nets. Among all models used in this study, the most successful model was the Ward nets, which consisted of three different architecture designs. The designs are:

1.1 Two hidden slabs with different activation functions.

1.2 Three hidden slabs with different activation functions.

1.3 Two hidden slabs with different activation functions and a jump connection.

Both the second and third architecture designs were found to be the best to use for the prediction of the three different parameters. Several models with different architecture design were used in this study to check their effective on the output. 
Based on the most effective models, number of runs were done to predict three different parameters, namely, bit type, ROP, and cost per foot. In general, the neural network architecture using the Ward nets with two hidden slabs and jump connection (1.3) design was found to be more effective in predicting the bit type than other architecture designs. At the same time, Ward nets with three hidden slabs (1.2) was found to be more effective in predicting both ROP and cost per foot than the network used for bit type prediction. In some runs both architectures yielded similar correlations.

In this study, the learning rates of 0.1 and 0.05 and momentum values of 0.1 and 0.5 were used in most of the runs. Learning rate establishes the speed of processing, momentum determines the capacity to over come punctual errors or scatter. Different learning rates and momentum have been used in this study. In most of the runs, the default values of both learning rate (0.1) and momentum (0.1) were used. In some cases both learning rate and momentum were adjusted to 0.05 and 0.5 , respectively. In this study, readjusting both learning rate and momentum found to have an impact in some of the result. In general, the $\mathrm{R}$ square values were improved.

Different numbers of input neurons were chosen in this study and the contribution factors for each input neuron on the output value was determined. For example Kuwait-1 (NN3) Run (3.C), a total of 13 input neurons were chosen to train them with the network to predict the cost per foot. The number of hidden neurons used in the design are based on the following formula yielding the default number of neurons:

Number of Neurons $=\frac{1}{2}[\{$ Number of Outputs $\}+\{$ Number of Inputs $\}]+\sqrt{[\text { Number of Patterns }]}$ 


\subsubsection{Training}

The training of the neural network can be accomplished by using the total pattern file or the training set selected using one of the criteria. All runs conducted in this study used the training set for neural network until the minimum error was observed for the test set. All results were evaluated based on the linear correlation coefficient, $\mathrm{R}$, and the coefficient of multiple determination, $\mathrm{R}^{2}$. The outputs from the neural network are plotted against the field-measured parameters.

The neural networks were developed to predict three drilling parameters, which are bit type, ROP, and cost per foot. Different sets of files from several drilling operations were used to predict outputs. The data gathered were in three main sets, which are Kuwait-1, Kuwait-2, and Kuwait-3 sets. Both Kuwait- 1 and Kuwait-2 sets contained the same drilling parameters, where Kuwait-3 set had less number of drilling parameters than both of Kuwait-1, and Kuwait-2 sets. For each set, eight extra coding parameters were added to the data set to represent different coding of the bit type. Eight different runs were then performed to predict the bit type, where each run used separate bit coding. This was done to choose the code that best represents the bit type among all of the eight codes.

In this study, the data gathered consisted of various drilling parameters and several runs were conducted to choose the most important parameters based on contributing factors to the output values. Among several runs with different files, five sets were then chosen to estimate the three-predictions. The final sets that were trained for the three-predictions are Kuwait-1, Kuwait-2, and Kuwait-3, which are the main sets. And two more subsets created for further analysis, which are Kuwait-4 and Kuwait-5. 
In summary, three different neural network models were developed for five different data sets representing unique drilling conditions. All of the runs are listed in Table-1 with the input and the output drilling parameters. 


\begin{tabular}{|c|c|c|c|}
\hline DATA SET & RUN & IINPUT & "OUTPUT \\
\hline Kuwait-1 & 1.A & $\begin{array}{l}\text { Bit size, total flow area (tfa), depth out, drilled interval, ROP, minimum WOB, } \\
\text { maximum WOB, minimum RPM, maximum RPM, flow rate (q). }\end{array}$ & Bit type \\
\hline Kuwait-1 & 1.B & $\begin{array}{l}\text { Bit type , bit size, tfa, depth out, drilled interval, hrs, minimum WOB, maximum } \\
\text { WOB, RPM min, RPM maximum, and q. }\end{array}$ & ROP \\
\hline Kuwait-1 & 1.C & $\begin{array}{l}\text { Bit type, bit size, tfa, depth out, drilled interval, hrs, min WOB, maximum } \\
\text { WOB, minimum RPM, maximum RPM, bit cost } \$ \text {, and q. }\end{array}$ & Cost per $\mathrm{ft}$ \\
\hline Kuwait-2 & 2.A & $\begin{array}{l}\text { Bit size, tfa, depth out, drilled interval, ROP, min WOB, maximum WOB, } \\
\text { minimum RPM, maximum RPM, and } \mathrm{q}\end{array}$ & Bit Type \\
\hline Kuwait-2 & 2.B & $\begin{array}{l}\text { Bit type , bit size, tfa, depth out, drilled interval, hrs, minimum WOB, maximum } \\
\text { WOB, minimum RPM, maximum RPM, and q. }\end{array}$ & ROP \\
\hline Kuwait-2 & 2.C & $\begin{array}{l}\text { Bit type, size, tfa, depth out, drilled interval, hrs, minimum WOB, maximum } \\
\text { WOB, min RPM, maximum RPM, bit cost } \$ \text {, and } \mathrm{q}\end{array}$ & Cost per $\mathrm{ft}$ \\
\hline Kuwait-3 & 3.A & Bit size, tfa, depth out, drilled interval, ROP, WOB, RPM, and flow rate (q). & Bit Type \\
\hline Kuwait-3 & 3.B & Bit type, bit size, tfa, depth out, drilled interval, hr, ROP, WOB, RPM, and q. & ROP \\
\hline Kuwait-3 & 3.C & Bit type, bit size, tfa, depth out, drilled interval, hr, WOB, RPM, q, and bit cost & Cost per $\mathrm{ft}$ \\
\hline Kuwait-4 & 4 & Bit size, tfa, depth out, drilled interval, ROP, WOB, RPM, and flow rate (q). & Bit type \\
\hline Kuwait-4 & 4.A & Bit size, tfa, depth out, drilled interval, ROP, WOB, RPM, and flow rate (q). & Bit type \\
\hline Kuwait-4 & 4.B & Bit type, bit size, tfa, depth out, drilled interval, hr, ROP, WOB, RPM, and q & ROP \\
\hline Kuwait-4 & 4.C & Bit type, bit size, tfa, depth out, drilled interval, hr, WOB, RPM, q, and bit cost & Cost per $\mathrm{ft}$ \\
\hline Kuwait-5 & 5.A & Bit size, tfa, depth out, drilled interval, ROP, WOB, RPM, and flow rate (q). & Bit Type \\
\hline Kuwait-5 & $5 . \mathrm{B}$ & Bit type, bit size, tfa, depth out, drilled interval, hr, ROP, WOB, RPM, and q. & ROP \\
\hline Kuwait-5 & 5.C & Bit type, bit size, tfa, depth out, drilled interval, hr, WOB, RPM, q, and bit cost & Cost per $\mathrm{ft}$ \\
\hline
\end{tabular}

Table 1. List of runs with input and output parameters. 


\section{RESULTS AND DISCUSSION}

Three different drilling parameters namely bit type (NN1), rate of penetration (NN2), and cost per foot (NN3) were estimated using the neural networks. Five different sets of data are used in this study, which are identified as Kuwait-1, Kuwait-2, Kuwait-3, Kuwait-4, and Kuwait-5. Both Kuwait-4 and Kuwait-5 are subsets created for further analysis of the threeparameter predictions. An artificial neural network was developed for each of the five data sets. The input and output parameters are listed in Table 1.

In this study most of the drilling parameters were used as inputs and runs were conducted to determine the contribution factor for each input parameter. The most important parameters, which have more contribution to the output value, found to be the following:

Bit type, size, total flow area, depth out, interval drilled, rotating hours, ROP, minimum WOB, maximum WOB, minimum RPM, maximum RPM, and flow rate. (Bit cost $\$$ when predicting $\$ / \mathrm{ft}$ )

Different types of architectural designs as well as different learning rates and momentums were used in this study to understand their effect and contribution to the final results. Among all models studied the most successful model for this study was the Ward nets. Ward nets model is based on different architecture designs with two or three hidden slabs. In general, the architecture design with two hidden slabs with different activation functions and a jump connection was found to be more effective in predicting the bit type. And the architecture design with three hidden slabs with different activation functions was found to be more effective in predicting both ROP and cost per foot. Additional models with different architecture designs other than Ward nets, were used to determine their efficiency in the prediction of the output. 
The range of the minimum and maximum values have an impact on the results, a good result can be achieved when the range of the minimum and maximum values are tight around. Each set contained different range of minimum and maximum values and the ranges are listed in Table 2, Table 3, Table 4, Table 5, and Table 6 for Kuwait-1, Kuwait-2, Kuwait-3, Kuwait-4, and Kuwait-5, respectively.

An artificial neural network was developed for each of the five sets used in this study. The results are shown in Kuwait-1, Kuwait-2, Kuwait-3, Kuwait-4, and Kuwait-5 sections. 


\subsection{Kuwait-1 Data Set.}

The result of the three drilling parameters for this data set are listed in Figure 3 for bit type prediction, Figure 4 for rate of penetration prediction, and Figure 5 for cost per foot prediction. The ranges of values for Kuwait-1 data set are listed in Table 2.

\begin{tabular}{||l|l|l||}
\hline DRILLING PARAMETERS & MINIMUM & MAXIMUM \\
\hline Bit type & 1 & 277 \\
\hline Bit size, (inch) & 4.5 & 28 \\
\hline Total flow area, (square inch) & 0.1534 & 1.491 \\
\hline Depth in, (feet) & 0 & 16204 \\
\hline Depth out, (feet) & 127 & 16463 \\
\hline Drilled interval, (feet) & 100 & 5105 \\
\hline Rotating hours & 1.5 & 175 \\
\hline Rate of penetration, (ft/hr) & 1 & 188 \\
\hline Minimum WOB, (1000 lbs) & 5 & 85 \\
\hline Maximum WOB, (1000 lbs) & 5 & 180 \\
\hline Minimum RPM, (rev/min) & 25 & 862.9 \\
\hline Maximum RPM, (rev/min) & 30 & \\
\hline Fit cost in dollars, (\$) & 73 & \\
\hline Cost per foot, (\$/ft) & 752.4 & \\
\hline
\end{tabular}

Table 2. The range of values for Kuwait-1 data set. 
Figure 3 shows the comparison of neural network predicted bit type with the selected bit type. The runs conducted with the final design for this model has produced linear correlation coefficient $(\mathrm{R})$ values ranging between 0.960 and 0.975 . Initially, 0.1 was used for the learning rate and momentum in the network and the correlation coefficient, $\mathrm{R}$, for the output was 0.960 . When both learning rate and momentum for this run were adjusted to 0.05 and 0.5 , respectively, the correlation coefficient value for the output improved from $R=0.960$ to $R=0.975$. The architecture design used for this prediction was the Ward nets with two hidden slabs with different activation functions and a jump connection (1.3). Other architecture designs were also used, however the correlation coefficient values were not as good as in architecture (1.3). For example, when the Standard Backpropagation (category 1) was used to predict the bit type, a correlation coefficient value of $\mathrm{R}=0.940$ was obtained. Also when the Jorda-Elman nets (category 2) was used, the correlation coefficient was 0.930 . The same inputs, output, learning rate and momentum with architecture (1.3) produced a correlation coefficient value of 0.975 . Thus architecture (1.3) was used to predict the bit type in all neural networks. Also the bit type prediction for Kuwait-1 has produced the best linear correlation coefficient value when compared to the rest of the results from other data sets.

The comparison of neural network predicted rate of penetration values with the field measured values are shown in Figure 4. The final design for this model has produced a linear correlation coefficient $(\mathrm{R})$ value equal to 0.990 . The learning rate and the momentum used in this run were 0.05 and 0.5 , respectively. The neural network architecture design used for this prediction was the Ward nets with three hidden slabs with different activation functions (1.2). Other architectural designs were also used, however, the correlation coefficient values were not as good as the values obtained by architecture (1.2). For example, the ROP prediction with the 
Standard Backpropagation architecture (category 1) yielded a correlation coefficient value of 0.862. Also when the Jorda-Elman nets architecture (category 2) was used to predict ROP a correlation coefficient value of 0.970 was obtained. With the same inputs, output, learning rate, and momentum, the network architecture (1.2) had a correlation coefficient value of 0.990 for ROP prediction. Thus architecture (1.2) was used to predict the bit type for all data sets.

Figure 5 compares the neural network predicted cost per foot values with the field measured values. The final design for this model has a linear correlation coefficient value of 0.989. The learning rate and the momentum used in this run were 0.05 and 0.5 , respectively. The architecture design used for this prediction was the Ward nets with three hidden slabs with different activation functions. The neural network architecture used to predict the rate of penetration was also used for the cost per foot predictions. 


\subsection{Kuwait-2 Data Set.}

The result of the three drilling parameters for this data set are listed in Figure 6 for bit type prediction, Figure 7 for rate of penetration prediction, and Figure 8 for cost per foot prediction. The ranges of values for Kuwait-2 data set are listed in Table 3 .

\begin{tabular}{||l|l|l||}
\hline DRILLING PARAMETERS & MINIMUM & MAXIMUM \\
\hline Bit type & 1 & 489 \\
\hline Bit size, (inch) & 4.125 & 36 \\
\hline Total flow area, (square inch) & 0.049 & 7.517 \\
\hline Depth out, (feet) & 104 & 20774 \\
\hline Drilled interval, (feet) & 100 & 8672 \\
\hline Rotating hours & 0.5 & 390.5 \\
\hline Rate of penetration, (ft/hr) & 1 & 448 \\
\hline Minimum WOB, (1000 lbs) & 1 & 80 \\
\hline Maximum WOB, (1000 lbs) & 2 & 100 \\
\hline Minimum RPM, (rev/min) & 15 & 259 \\
\hline Maximum RPM, (rev/min) & 15 & 259 \\
\hline Flow rate, (gallons/min) & 23 & 1900 \\
\hline Cost per foot, (\$/ft) & 2.31 & 832.9 \\
\hline \hline
\end{tabular}

Table 3. The range of values for Kuwait-2 data set. 
Figure 6 shows the comparison of neural network predicted bit type with the selected bit type. The final design for this model has a linear correlation coefficient value of 0.868 . The architecture design used for this prediction was the Ward nets with two hidden slabs with different activation functions and a jump connection. The learning rate and the momentum used for this run were 0.05 and 0.5 , respectively.

The comparison of neural network predicted rate of penetration values with the field measured values are shown in Figure 7. The final design for this model has a linear correlation coefficient value of 0.960 . The learning rate and the momentum used for this run were 0.05 and 0.5 , respectively. The architecture design used for this prediction was the Ward nets with three hidden slabs with different activation functions.

The neural network predicted cost per foot values are compared with the field measured values in Figure 8. The final design for this model has a linear correlation coefficient value of 0.947. The learning rate and the momentum used for this run were 0.05 and 0.5 , respectively. The architecture design used for this prediction was the Ward nets with three hidden slabs with different activation functions. 


\subsection{Kuwait-3 Data Set.}

The result of the three drilling parameters for this data set are listed in Figure 9 for bit type prediction, Figure 10 for rate of penetration prediction, and Figure 11 for cost per foot prediction. The ranges of values for Kuwait- 3 data set are listed in Table 4 .

\begin{tabular}{||l|l|l||}
\hline DRILLING PARAMETERS & MINIMUM & MAXIMUM \\
\hline Bit type & 1 & 405 \\
\hline Bit size, (inch) & 6.125 & 28 \\
\hline Total flow area, (square inch) & 0.173 & 2.506 \\
\hline Depth out, (feet) & 104 & 17892 \\
\hline Drilled interval, (feet) & 100 & 5833 \\
\hline Rotating hours & 0.5 & 420 \\
\hline Rate of penetration, (ft/hr) & 2.32 & 448 \\
\hline WOB, (1000 lbs) & 2 & 83 \\
\hline RPM, (rev/min) & 11 & 315 \\
\hline Flow rate, (gallons/min) & 10 & 1297 \\
\hline Cost per foot, (\$/ft) & 2.31 & 568.67 \\
\hline \hline
\end{tabular}

Table 4. The range of values for Kuwait-3 data set. 
The comparison of neural network predicted bit type with the selected bit type is given in Figure 9. In this set, bit type code number 6, V2, was used as the output value that represent the bit type. The final design for this model has a linear correlation coefficient value of 0.879 . The learning rate and the momentum used for this run were 0.05 and 0.5 , respectively. The architecture design used for this prediction was the Ward nets with two hidden slabs with different activation functions and a jump connection.

Figure 10 shows the comparison of neural network predicted rate of penetration values with the field measured values. The final design for this model has a linear correlation coefficient value of 0.973 . The learning rate and the momentum used for this run were 0.05 and 0.5 , respectively. The architecture design used for this prediction was the Ward nets with three hidden slabs with different activation functions.

The comparison of neural network predicted cost per foot values with the field measured values are shown in Figure 11. The final design for this model has produced a linear correlation coefficient value of 0.990 . The learning rate and the momentum used for this run were 0.05 and 0.5 , respectively. The architecture design used for this prediction was the Ward nets with three hidden slabs with different activation functions. 


\subsection{Kuwait-4 Data Set.}

The result of the three drilling parameters for this data set are listed in Figure 12 and Figure 13 for bit type prediction, Figure 14 for rate of penetration prediction, and Figure 15 for cost per foot prediction. The ranges of values for Kuwait-4 data set are listed in Table 5 .

\begin{tabular}{||l|l|l||}
\hline DRILLING PARAMETERS & MINIMUM & MAXIMUM \\
\hline Bit type & 1 & 106 \\
\hline Bit size, (inch) & 6.125 & 26 \\
\hline Total flow area, (square inch) & 0.314 & 2.356 \\
\hline Depth out, (feet) & 950 & 10660 \\
\hline Drilled interval, (feet) & 105 & 4806 \\
\hline Rotating hours & 1 & 156.5 \\
\hline Rate of penetration, (ft/hr) & 3.28 & 275.56 \\
\hline WOB, (1000 lbs) & 4 & 65 \\
\hline RPM, (rev/min) & 40 & 180 \\
\hline Flow rate, (gallons/min) & 96 & 313.22 \\
\hline Cost per foot, (\$/ft) & 6.47 & \\
\hline \hline
\end{tabular}

Table 5. The range of values for Kuwait-4 data set. 
Figure 12 and Figure 13 show the comparison of neural network predicted bit type with the selected bit type for different bit type code. In this subset, both of the bit type coding number 6 (V2) and number 9 (bit code) were used to estimate as the output value separately. The final design for this model has a linear correlation coefficient value of 0.947 for V2 code, and a linear correlation coefficient value of 0.936 for bit coding number 9 . The learning rate and the momentum used for the runs were 0.05 and 0.5 , respectively. The architecture design used for this prediction was the Ward nets with two hidden slabs with different activation functions and a jump connection.

The comparison of neural network predicted rate of penetration values with the field measured values are shown in Figure 14. The final design for this model has a linear correlation coefficient value of 0.983 . The learning rate and the momentum used for this run were 0.05 and 0.5 , respectively. The architecture design used for this prediction was the Ward nets with three hidden slabs with different activation functions.

Figure 15 shows the comparison of neural network predicted cost per foot values with the field measured values. The final design for this model has produced a linear correlation coefficient value of 0.963 . The learning rate and the momentum were 0.05 and 0.5 , respectively. The architecture design used for this prediction was the Ward nets with three hidden slabs with different activation functions. 


\subsection{Kuwait-5 Data Set.}

The result of the three drilling parameters for this data set are listed in Figure 16 for bit type prediction, Figure 17 for rate of penetration prediction, and Figure 18 for cost per foot prediction. The range of values for Kuwait-5 data set are listed in Table 6 .

\begin{tabular}{||l|l|l||}
\hline DRILLING PARAMETERS & MINIMUM & MAXIMUM \\
\hline Bit type & 1 & 129 \\
\hline Bit size, (inch) & 6.125 & 28 \\
\hline Total flow area, (square inch) & 0.24 & 1.335 \\
\hline Depth out, (feet) & 340 & 14000 \\
\hline Drilled interval, (feet) & 100 & 5550 \\
\hline Rotating hours & 1.5 & 140 \\
\hline Rate of penetration, (ft/hr) & 2.45 & 114 \\
\hline WOB, (1000 lbs) & 5 & 70 \\
\hline RPM, (rev/min) & 33 & 190 \\
\hline Flow rate (gallons/min) & 130 & 1200 \\
\hline Cost per foot, (\$/ft) & 10.22 & 438.17 \\
\hline \hline
\end{tabular}

Table 6. The range of values for Kuwait-5 data set. 
Figure 16 shows the comparison of neural network predicted bit type with the selected bit type. The final design for this model has a linear correlation coefficient value of 0.903 . The learning rate and the momentum used for this run were 0.05 and 0.5 , respectively. The architecture design used for this prediction was the Ward nets with two hidden slabs with different activation functions and a jump connection.

The comparison of neural network predicted rate of penetration values with the field measured values are given in Figure 17. The final design for this model has a linear correlation coefficient value of 0.989 . The learning rate and the momentum used for this run were 0.05 and 0.5 , respectively. The architecture design used for this prediction was the Ward nets with three hidden slabs with different activation functions.

Figure 18 shows the comparison of neural network predicted cost per foot values with the field measured values. The final design for this model has produced a linear correlation coefficient value of 0.975 . The learning rate and the momentum were 0.05 and 0.5 , respectively. The architecture design used for this prediction was the Ward nets with three hidden slabs with different activation functions. 


\subsection{SUMMARY}

In summary a comparison for the results estimated are shown in for bit type prediction, rate of penetration prediction, and cost per foot prediction in Table 7, Table 8, and Table 9, respectively. The results show good predictions of the bit type, the ROP, and the cost-per-foot for the data sets used in this study.

Kuwait-1 data set was found to produce a better correlation coefficient for the bit type prediction than the rest of the sets. One of the main factors that altered the bit type prediction is the large number of the bits that have been used in this study. As the number of bits increased, the system had fewer amounts of data that contribute to each bit type, which lead to less improvement in the linear correlation coefficient, R. When reducing the number of data by using only information from a region or field such as Kuwait-4 and Kuwait-5, the output value did show improvement.

Based on the preliminary runs, the bit types coded in the data sets were found to be ineffective in describing all bit types. Therefore, nine different bit codes were developed in this study. The main reason for that was to overcome and reduce the factors that alter the bit type prediction. Among all of the nine different codes, both of code (6) and (9) were found to be the most effective. Code 6, which is called (V2), was created to limit the data and to make a variation between the different type of bits. One problem with this type of coding is that one code will have several bit type, it does not correspond to a specific bit type. For example, Kuwait-3 and Kuwait-4 were used to predict the bit type using this specific type of code. Code 9, which is called bit code, yielded the best correlation among all data sets including code 6 . The advantage of this type of coding is the assignment of a number for each bit. 
Kuwait-4 was specifically created to predict all of the three drilling parameters for one region. This subset consists of two different fields located in one area. Run with Kuwait-4 data set has produced better correlation when the bit was the predicted output value. For example, the bit type prediction for Kuwait-3 data set has produced a linear correlation coefficient value of 0.879 , where the same prediction for Kuwait-4 data set has produced 0.947. The (V2) code was used in Kuwait-3 and Kuwait-4 data sets to predict the bit type.

Another subset, Kuwait-5 was created to analyse only one field data. The purpose of this specific set is to test the neural networks for predicting three different drilling parameters with data obtained from only one field with same characteristics. Even though fewer data were used compared to other data sets, the one field data have been found to give good results in predicting all of the three-parameters. In this set code (bit code) was used to predict the bit type.

Other than the bit type, the rest of the predictions, ROP and cost-per-foot found to produce good results for all of the sets. 


\begin{tabular}{|c|c|c|c|}
\hline $\begin{array}{l}\text { NAME OF } \\
\text { DATA SET }\end{array}$ & $\begin{array}{l}\text { NUMBER } \\
\text { OF DATA }\end{array}$ & $\begin{array}{l}\text { CORRELATION } \\
\text { COEFFICIENT }\end{array}$ & INPUT PARAMETERS \\
\hline Kuwait-1 & 1893 & 0.975 & $\begin{array}{l}\text { Bit size, total flow area, depth out, drilled interval, ROP, } \\
\text { minimum WOB, maximum WOB, minimum RPM, } \\
\text { maximum RPM, and flow rate (q). }\end{array}$ \\
\hline Kuwait-2 & 4466 & 0.868 & $\begin{array}{l}\text { Bit size, total flow area, depth out, drilled interval, ROP, } \\
\text { minimum WOB, maximum WOB, minimum RPM, } \\
\text { maximum RPM, and flow rate (q). }\end{array}$ \\
\hline Kuwait-3 & 2392 & 0.879 & $\begin{array}{l}\text { Bit size, total flow area, depth out, drilled interval, ROP, } \\
\text { WOB, RPM, and flow rate. }\end{array}$ \\
\hline $\begin{array}{c}\text { Kuwait-4 } \\
\text { For (V2 code) }\end{array}$ & 450 & 0.947 & $\begin{array}{l}\text { Bit size, total flow area, depth out, drilled interval, ROP, } \\
\text { WOB, RPM, and flow rate. }\end{array}$ \\
\hline $\begin{array}{l}\text { Kuwait-4-A } \\
\text { For (bit code) }\end{array}$ & 450 & 0.936 & $\begin{array}{l}\text { Bit size, total flow area, depth out, drilled interval, ROP, } \\
\text { WOB, RPM, and flow rate. }\end{array}$ \\
\hline Kuwait-5 & 367 & 0.903 & $\begin{array}{l}\text { Bit size, total flow area, depth out, drilled interval, ROP, } \\
\text { WOB, RPM, and flow rate. }\end{array}$ \\
\hline
\end{tabular}

Table 7. Comparison of results for bit type predictions. 


\begin{tabular}{|c|c|c|c|}
\hline $\begin{array}{l}\text { NUMBER OF } \\
\text { DATA SET }\end{array}$ & $\begin{array}{l}\text { NUMBER } \\
\text { OF DATA }\end{array}$ & $\begin{array}{l}\text { CORRELATION } \\
\text { COEFFICIENT }\end{array}$ & INPUT PARAMETERS \\
\hline Kuwait-1 & 1893 & 0.990 & $\begin{array}{l}\text { Bit type, bit size, total flow area, depth out, drilled } \\
\text { interval, rotating hours, minimum WOB, maximum } \\
\text { WOB, minimum RPM, maximum RPM, and flow rate. }\end{array}$ \\
\hline Kuwait-2 & 4466 & 0.960 & $\begin{array}{l}\text { Bit type, bit size, total flow area, depth out, drilled } \\
\text { interval, rotating hours, minimum WOB, maximum } \\
\text { WOB, minimum RPM, maximum RPM, and flow rate. }\end{array}$ \\
\hline Kuwait-3 & 2392 & 0.973 & $\begin{array}{l}\text { Bit type, bit size, total flow area, depth out, drilled } \\
\text { interval, rotating hours, ROP, WOB, RPM, and flow } \\
\text { rate. }\end{array}$ \\
\hline Kuwait-4 & 450 & 0.983 & $\begin{array}{l}\text { Bit size, total flow area, depth out, drilled interval, } \\
\text { ROP, WOB, RPM, and flow rate. }\end{array}$ \\
\hline Kuwait-5 & 367 & 0.989 & $\begin{array}{l}\text { Bit type, bit size, total flow area, depth out, drilled } \\
\text { interval, rotating hours, ROP, WOB, RPM, and flow } \\
\text { rate. }\end{array}$ \\
\hline
\end{tabular}

Table 8. Comparison of results for ROP predictions. 


\begin{tabular}{|c|c|c|c|}
\hline $\begin{array}{l}\text { NUMBER OF } \\
\text { DATA SET }\end{array}$ & $\begin{array}{l}\text { NUMBER } \\
\text { OF DATA }\end{array}$ & $\begin{array}{l}\text { CORRELATION } \\
\text { COEFFICIENT }\end{array}$ & $\begin{array}{l}\text { INPUT PARAMETERS } \\
\end{array}$ \\
\hline Kuwait-1 & 1893 & 0.989 & $\begin{array}{l}\text { Bit type, bit size, total flow area, depth out, drilled } \\
\text { interval, rotating hours, minimum WOB, maximum } \\
\text { WOB, minimum RPM, maximum RPM, bit cost \$, and } \\
\text { flow rate. }\end{array}$ \\
\hline Kuwait-2 & 4466 & 0.947 & $\begin{array}{l}\text { Bit type, bit size, total flow area, depth out, drilled } \\
\text { interval, rotating hours, minimum WOB, maximum } \\
\text { WOB, minimum RPM, maximum RPM, bit cost \$, and } \\
\text { flow rate. }\end{array}$ \\
\hline Kuwait-3 & 2392 & 0.990 & $\begin{array}{l}\text { Bit type, bit size, total flow area, depth out, drilled } \\
\text { interval, rotating hours, WOB, RPM, flow rate, and bit } \\
\text { cost } \$ \text {. }\end{array}$ \\
\hline Kuwait-4 & 450 & 0.963 & $\begin{array}{l}\text { Bit size, total flow area, depth out, drilled interval, } \\
\text { ROP, WOB, RPM, and flow rate. }\end{array}$ \\
\hline Kuwait-5 & 367 & 0.975 & $\begin{array}{l}\text { Bit type, bit size, total flow area, depth out, drilled } \\
\text { interval, rotating hours, WOB, RPM, flow rate, and bit } \\
\text { cost } \$ \text {. }\end{array}$ \\
\hline
\end{tabular}

Table 9. Comparison of results for cost per foot predictions. 


\section{CONCLUSIONS}

In this study artificial neural networks are used successfully to predict the drilling parameters. Three models were developed to predict the bit type, the rate of penetration (ROP), and the cost per foot, respectively. The three drilling models were tested with data from fields located in Kuwait. Five different sets of data used in this study, which are Kuwait-1, Kuwait-2, Kuwait-3, Kuwait-4, and Kuwait-5 sets.

The neural network models developed had correlation coefficient $(\mathrm{R})$ values ranging between [0.868] and [0.975] for bit type prediction, between [0.960] and [0.990] for rate of penetration prediction, and between [0.947] and [0.990] for cost per foot prediction. In general, the highest correlation coefficient values were obtained by Kuwait-1 data set.

Neural network architecture with two hidden slabs having different activation functions and a jump connection was found as the most effective design for bit type prediction. At the same time, Ward nets with three hidden slabs having different activation functions and a jump connection was found to be the most effective design for rate of penetration and cost per foot predictions.

The most important contributing factors for bit type prediction were bit size, total flow area, and minimum WOB for Kuwait-1; bit size and depth out for Kuwait-2; depth out for Kuwait-3; bit size and total flow area for Kuwait-4; and bit size, total flow area, depth out, and hours for Kuwait-5 data set. For the rate of penetration prediction the most important contributing factors were drilling hours and drilled interval for Kuwait-1; drilling hours and minimum WOB for Kuwait-2; drilling hours for Kuwait-3; drilled interval and drilling hours for Kuwait-4; and drilling hours, bit size, and drilled interval for Kuwait-5. For the cost per foot prediction the most important contributing factors were drilled interval for Kuwait-1; bit code 
and bit cost for Kuwait-2; drilled interval, RPM, and bit cost for Kuwait-3; drilled interval and bit cost for Kuwait-4, and drilled hours and bit size for Kuwait-5.

Results show that the bit type, ROP, and cost-per-foot can be estimated effectively for the new well to be drilled. This can be predicted with models developed by neural networks. Prediction of drilling parameters with the new developed method would decrease the percentage of trial and error resulting in cost savings. 


\section{REFERENCES}

Alsaleh, A., "Bit Optimization in Kuwait." Paper SPE 57552, presented at the SPE/IADC Middle East Drilling Technology Conference, Abu Dhabi, UAE, Nov 1999

Altmis, U., "Estimation of Drilling Parameters Using Neural Networks." MS Thesis, West Virginia University, Morgantown, WV, 1996.

Azar, J., "Drilling Engineering Mechanics.” U Tulsa; Tulsa, 1995.

Baker Hughes. "Drilling Bits." 1996. <www.bakerhughes.com/bakerhughes/bhidefil.htm>.

Bilgesu, H.I., Tetrick, L.T., Altmis, U., Mohaghegh, S., Ameri, S., "A New Approach for Prediction of Rate of Penetration (ROP) Values." paper SPE 39231, presented at the SPE Eastern Regional Meeting, Lexington, KY, Oct 1997.

Bilgesu, H.I., Altmis, U., Mohaghegh, S., Ameri, S., and Aminian, K., "A New Approach to Predict Bit Life based on Tooth and Bearing Failure." paper SPE 51082, presented at the SPE Eastern Regional Meeting, Pittsburgh, PA, November 9-21, 1998.

Bourgoyne, A.T.[et al.].,"Applied Drilling Engineering." Society of Petroleum Engineering, Richardson, TX, 1986.

“Drilling Hydraulics.” Hughes Christensen Co; Baker Hughes, Houston, TX, 1996.

Fausett, Laurene., "Fundamentals of Neural Networks." Prentice Hall, Englewood Cliffs, NJ, 1994.

Gabolde, Gilles, and Jean Ngyen, "Drilling Data Handbook”, Paris: Edition Technip, 1999.

IADC, "Drill Bits." 1999. <www.iadc.rg/drillbits.htm>.

Leecraft, J., "Rotary Drilling." Petroleum Extension Service, Austin, TX, 1990.

Security DBS, "Security DBS Bits." 1999. <www..securitydbs.com/index.html>.

Smith Bits, "Smith Bits Catalog." 27 July, 1998. <www.smithbits.com/>.

U.S. Energy Information Administration: "Kuwait." Oct. 1999. <www.eia.doe.gov/emeu/cabs/kuwait.html>. 
White, A. C., Molnar, D., Aminian, K., Mohaghegh, S., Ameri, S., Esposito, P., "The Application of ANN for Zone Identification in a Complex Reservoir." paper SPE 30977, presented at the SPE meeting, Morgantown, WV, Sep, 1995.

“1999 Drill Bit Classifier.” World Oil, 85, Sep. 1999. 


\section{APPENDIX [A]}

\section{Kuwait}

Kuwait contains 96.5 billion barrels of proven oil reserves (including its share of the Neutral Zone), or roughly 9\% of the world's total oil reserves. Along with Saudi Arabia and the United Arab Emirates, Kuwait remains one of the few oil producing countries with significant excess oil production capacity.

Kuwait contains an estimated 94 billion barrels of proven oil reserves, more than $9 \%$ of the world total. The Neutral Zone area, which Kuwait shares with Saudi Arabia, holds an additional 5 billion barrels of reserves, half of which belong to Kuwait. Most of Kuwait's oil reserves are located in the 70-billion barrel Greater Burgan area, which comprises the Burgan, Magwa and Ahmadi structures. Greater Burgan is widely considered the world's second largest oil field, surpassed only by Saudi Arabia's Ghawar field.

Kuwait's Raudhatain, Sabriya, and Minagish fields have large proven reserves as well, with 6 billion, 3.8 billion, and 2 billion barrels of oil, respectively. All of these fields have been producing since the 1950s. They generally contain medium to light crude oil with gravities in the $30^{\circ}-36^{\circ}$ API range. The South Magwa field, discovered in 1984, is estimated to hold at least 25 billion barrels of light crude oil with a $35^{\circ}-40^{\circ}$ API gravity. The Neutral Zone encompasses a 6,200 square-mile area partitioned equally between Kuwait and Saudi Arabia under a 1992 agreement. The Neutral Zone contains an estimated 5 billion barrels of oil and 1 trillion cubic feet (Tcf) of natural gas.

Another Kuwaiti field, Ratqa, has been the subject of controversy. Once thought to be an independent reservoir, Ratqa is actually a southern extension of Iraq's supergiant Rumaila field. During the weeks preceding Iraq's August 1990 invasion of Kuwait, Iraq had accused 
Kuwait of stealing billions of dollars worth of Rumaila oil, and had refused to negotiate a sharing or joint development arrangement for Ratqa and southern Rumaila. In its 1994 recognition of Kuwait's border, Iraq acknowledged a 1,919-foot extension of Ratqa further to the north.

\section{Geological Background}

The Cretaceous and Tertiary sequence primary consist of normally pressured sands, shales, and limestones. The shale intervals are highly reactive and require inhibited muds to prevent hydration and soulghing. The Jurassic sequence is made up of formations that include fractured oolitic limestone, salts, anhydrites, and shale. (Alsaleh, 1999)

\section{Current Oil Production}

The bulk of Kuwait's oil production occurs at the onshore Greater Burgan field, whose Burgan, Magwa, and Ahmadi structures produce roughly 1.6 million barrels per day (bbl/d) combined. Most of Kuwait's other producing fields are relatively small and include the 250,000-bbl/d Raudhatain, 160,000-bbl/d Sabriya, 60,000-bbl/d Minagish, and 60,000-bbl/d Umm Gudair fields.

Construction of new gathering centers is a major focus of Kuwait's upstream capacity expansion program. Prior to the Iraqi invasion in 1990, Kuwait had 26 gathering centers (GCs), with a total capacity of 4 million bbl/d. All 26 GCs were either damaged or destroyed during the war. By 1993, 18 GCs had been restored. In January 1996, KPC awarded China Petroleum Engineering Construction Corporation (CPECC) a \$390-million contract to build two new GCs, a significant step in Kuwait's efforts to increase its oil production. CPECC is constructing the GCs, designated GC-27 and GC-28, at the Minagish and Umm Gudair fields. The work was to have been completed in 1998, but construction delays have pushed the anticipated completion 
date back to late 1999. When completed, these GCs are expected to boost capacity at the two western fields to more than 500,000 bbl/d. (US Energy Information Administration., 1999)

\section{Energy Overview}

\begin{tabular}{||ll||}
\hline PROVEN OIL RESERVES (1/1/99): & 96.5 BILLION BARRELS (INCLUDING 2.5 \\
& BILLION IN THE NEUTRAL ZONE, ALSO \\
& KNOWN AS THE DIVIDED ZONE OR \\
& PARTITIONED ZONE) \\
& \\
\hline $\begin{array}{l}\text { OPEC Crude Oil Production Quota } \\
\text { (since April 1, 1999): }\end{array}$ & 1.836 million bbl/d \\
\hline Oil Production Capacity (1999): & 2.4 million bbl/d (includes Neutral Zone) \\
\hline Oil Production (1999): & 2.0 million bbl/d (includes Neutral Zone) \\
\hline Oil Consumption (1999): & 146,000 bbl/d \\
\hline Crude Oil Refining Capacity (1999): & 886,000 bbl/d \\
\hline Major Crude Oil Customers: & Asia (around 60\%); Europe, South Africa, US \\
\hline Natural Gas Reserves (1/1/99): & 52.7 trillion cubic feet (Tcf) (includes Neutral Zone) \\
\hline Natural Gas Production (1997): & 0.33 Tcf \\
\hline Natural Gas Consumption (1997): & 0.33 Tcf \\
\hline Electric Generation Capacity (1/1/97): 7.0 gigawatts \\
\hline Electricity Production (1997): & 25 billion kilowatthours \\
\hline
\end{tabular}

Table 10. Kuwait Energy Overview. 


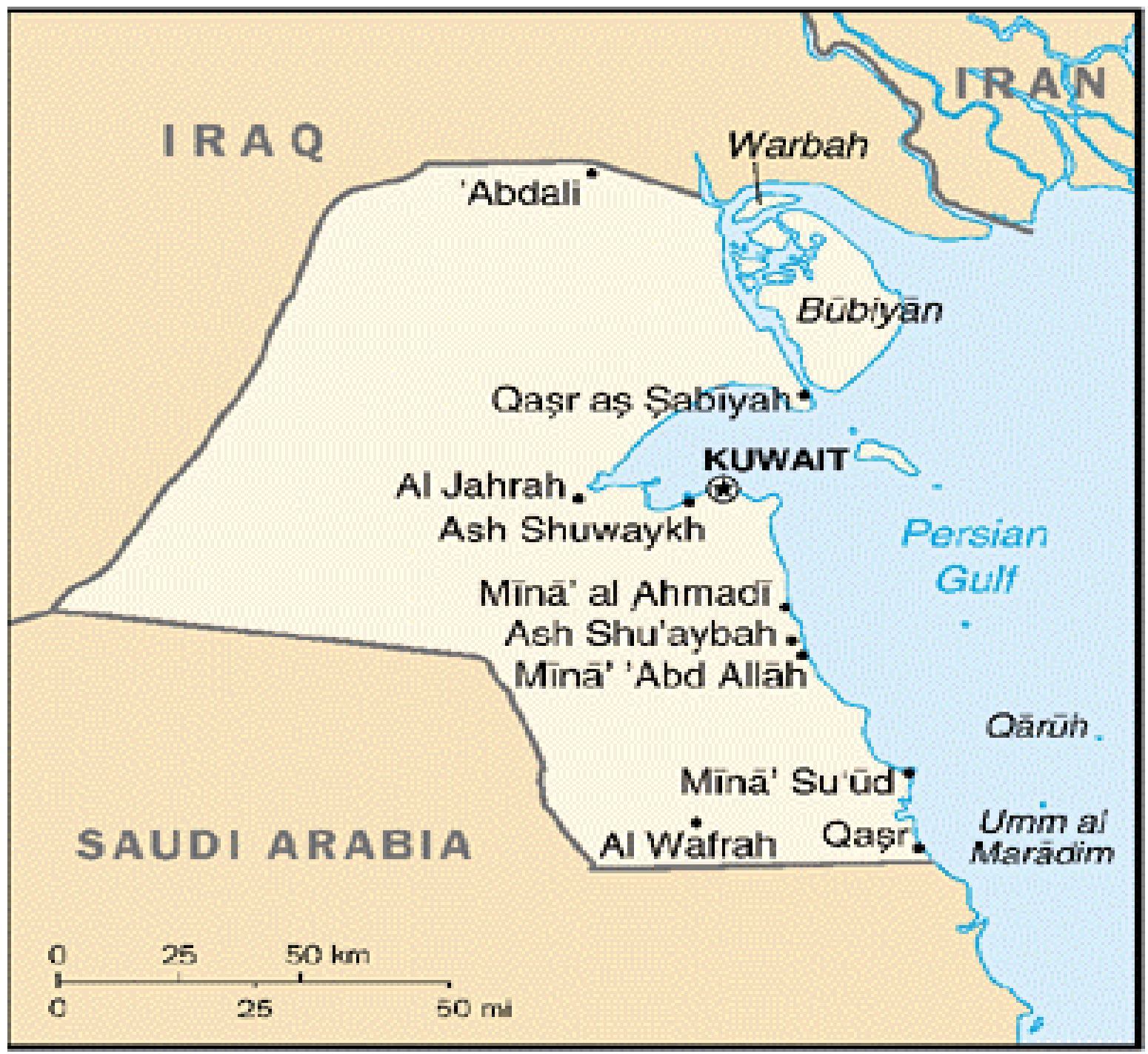

Figure 1. Kuwait Map. 


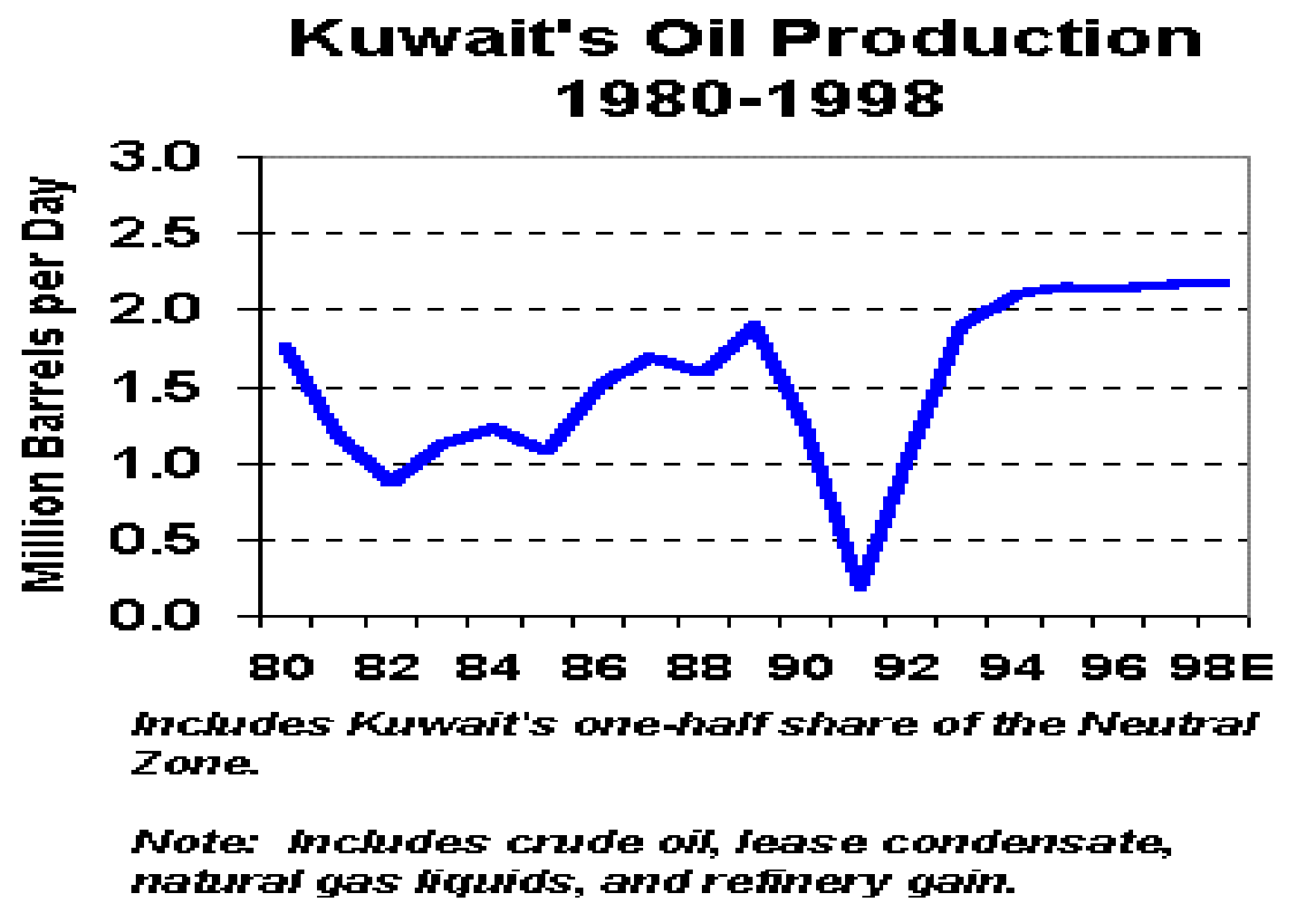

Figure 2. Kuwait oil production history.[1980-1998]. 


\section{APPENDIX [B]}

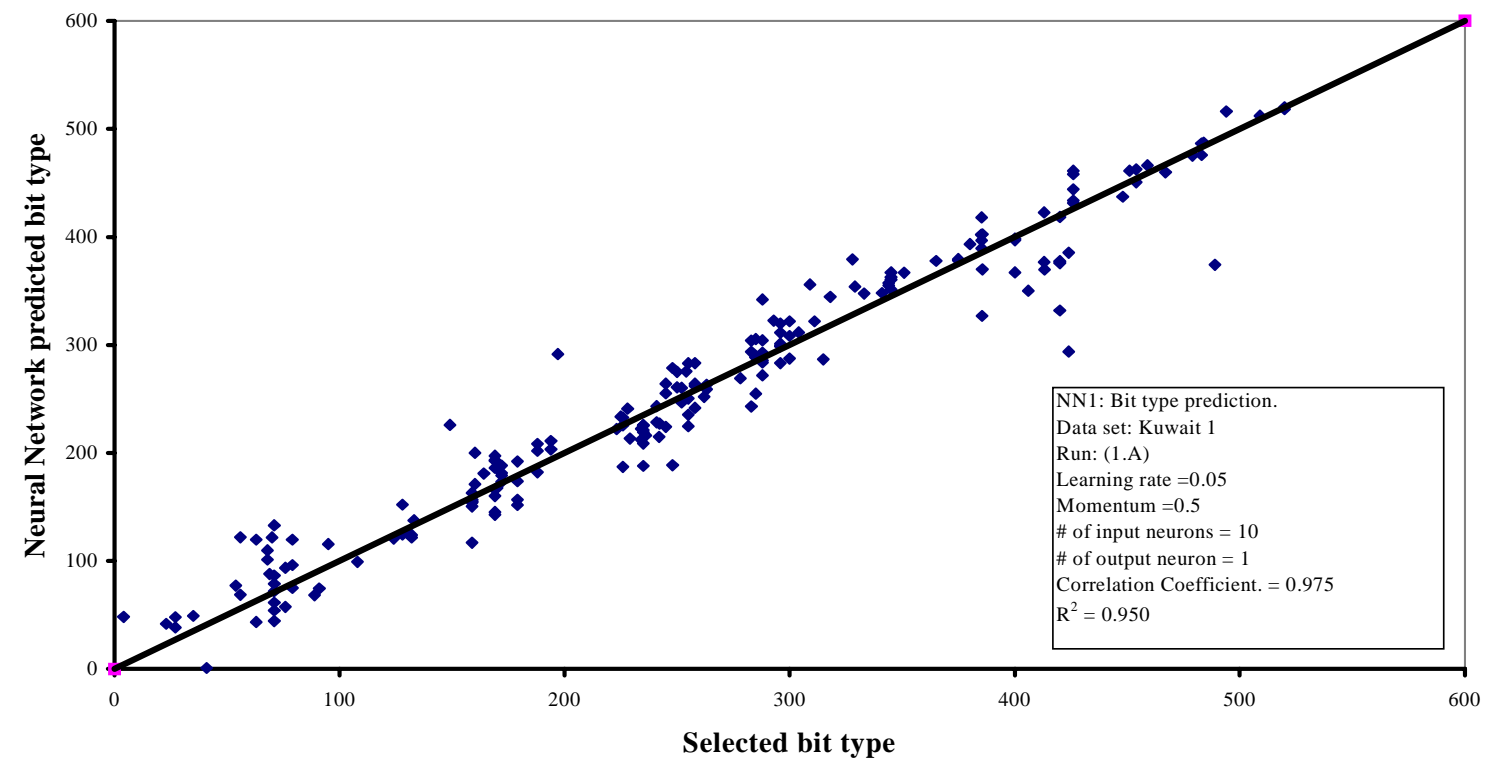

Figure 3. Comparison of neural network predicted bit type with the selected bit type. Kuwait-1 NN1 Run 1.A.

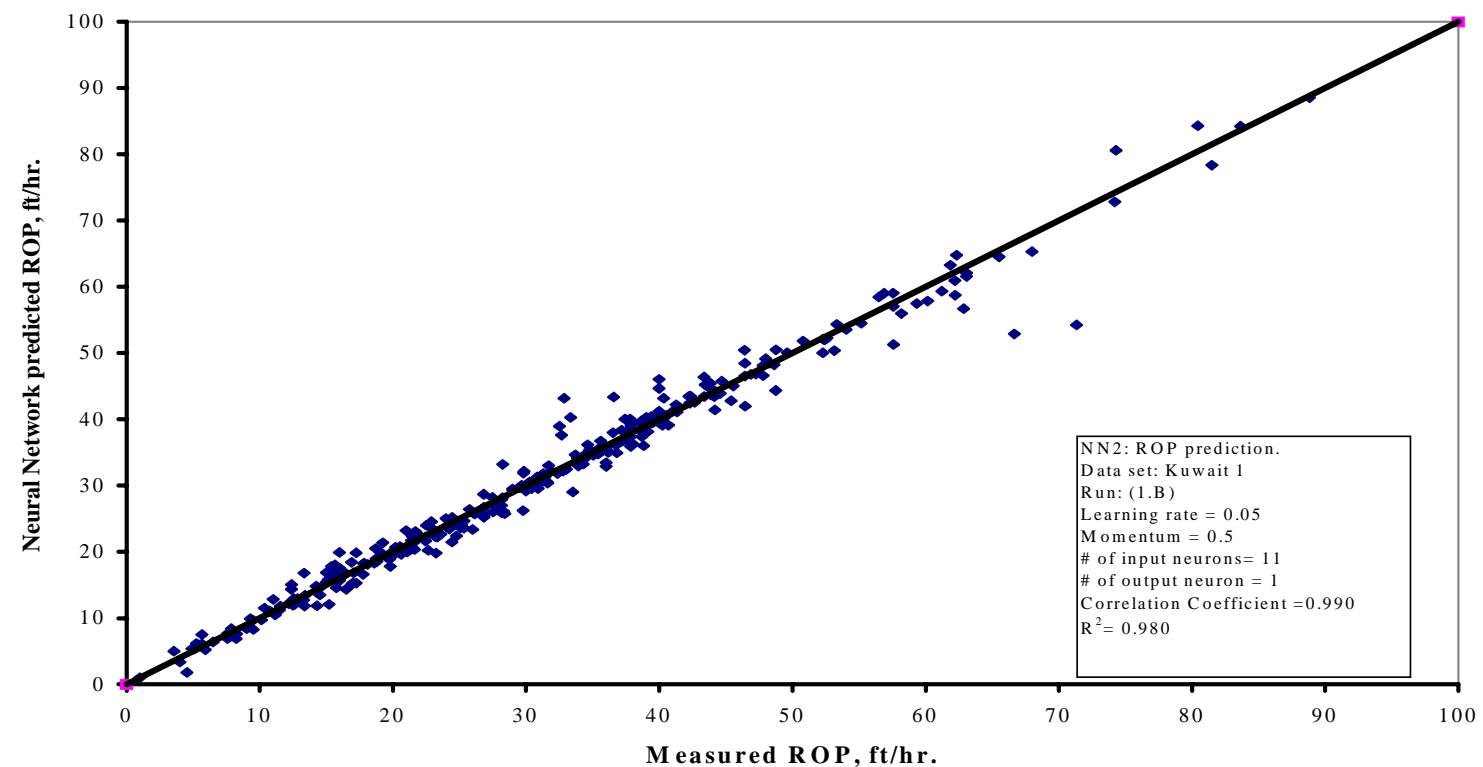

Figure 4. Comparison of neural network predicted ROP with the measured ROP. Kuwait-1 NN2 Run 1.B. 


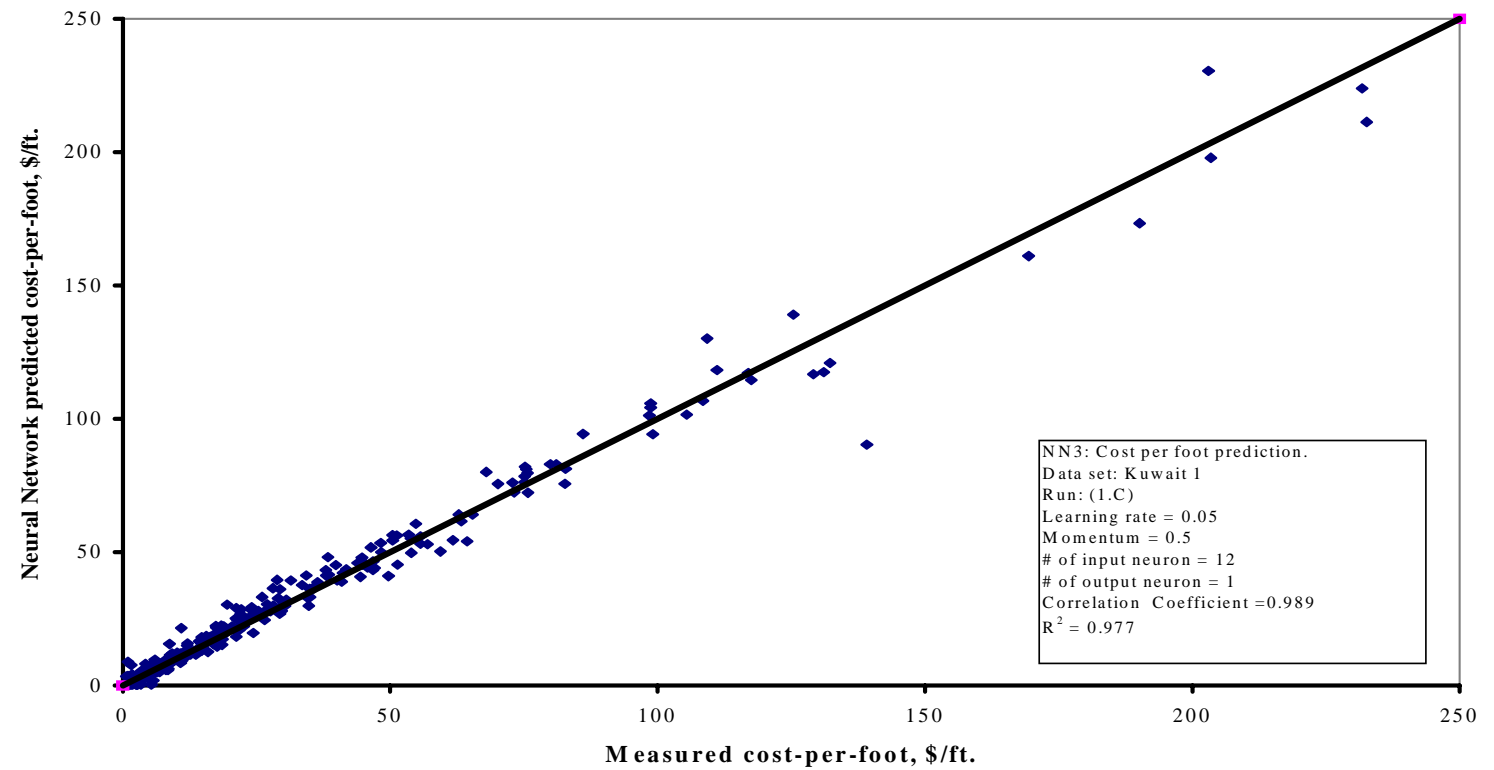

Figure 5. Comparison of neural network predicted cost per foot with the measured cost per foot. Kuwait-1 NN3 Run (1.C).

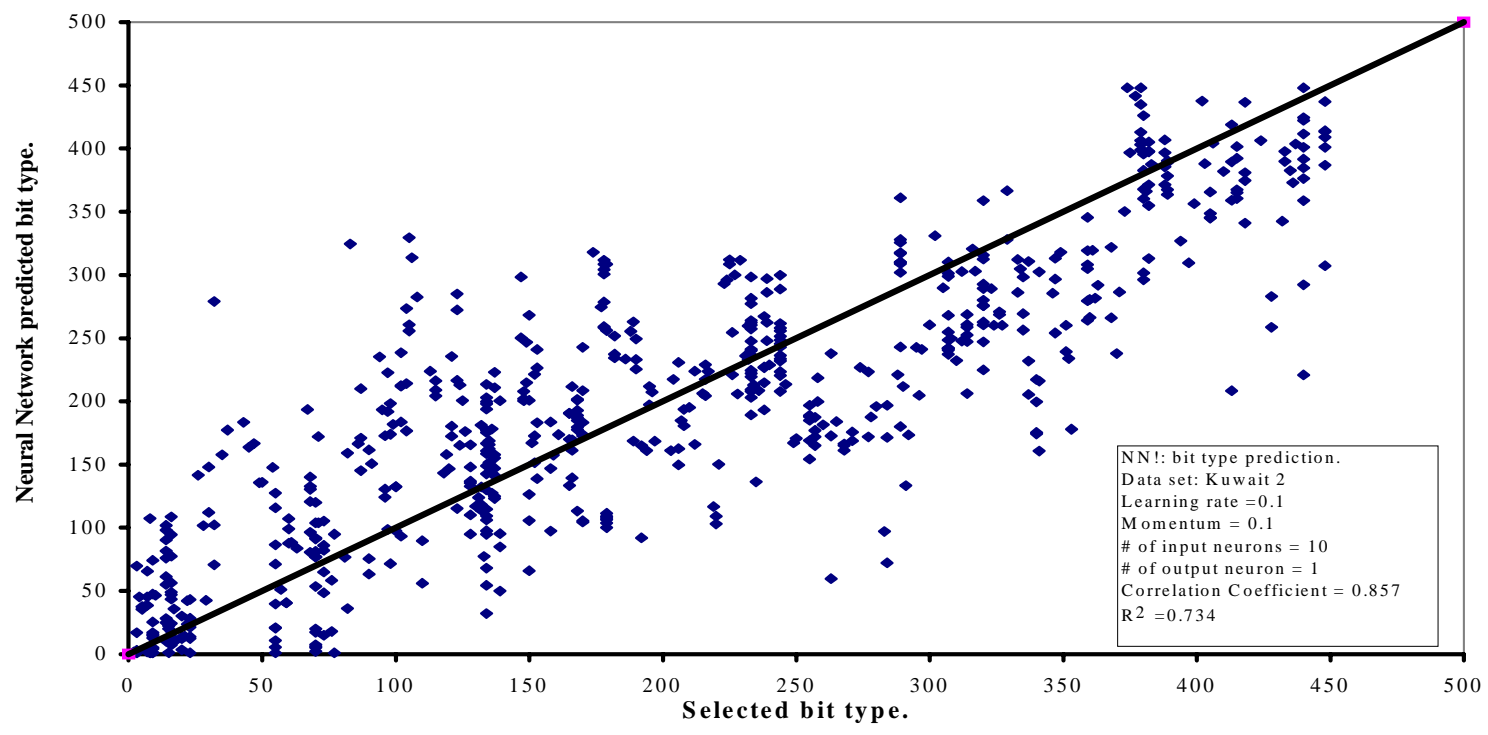

Figure 6. Comparison of neural network predicted bit type with the selected bit type. Kuwait-2 NN1 Run 2.A. 


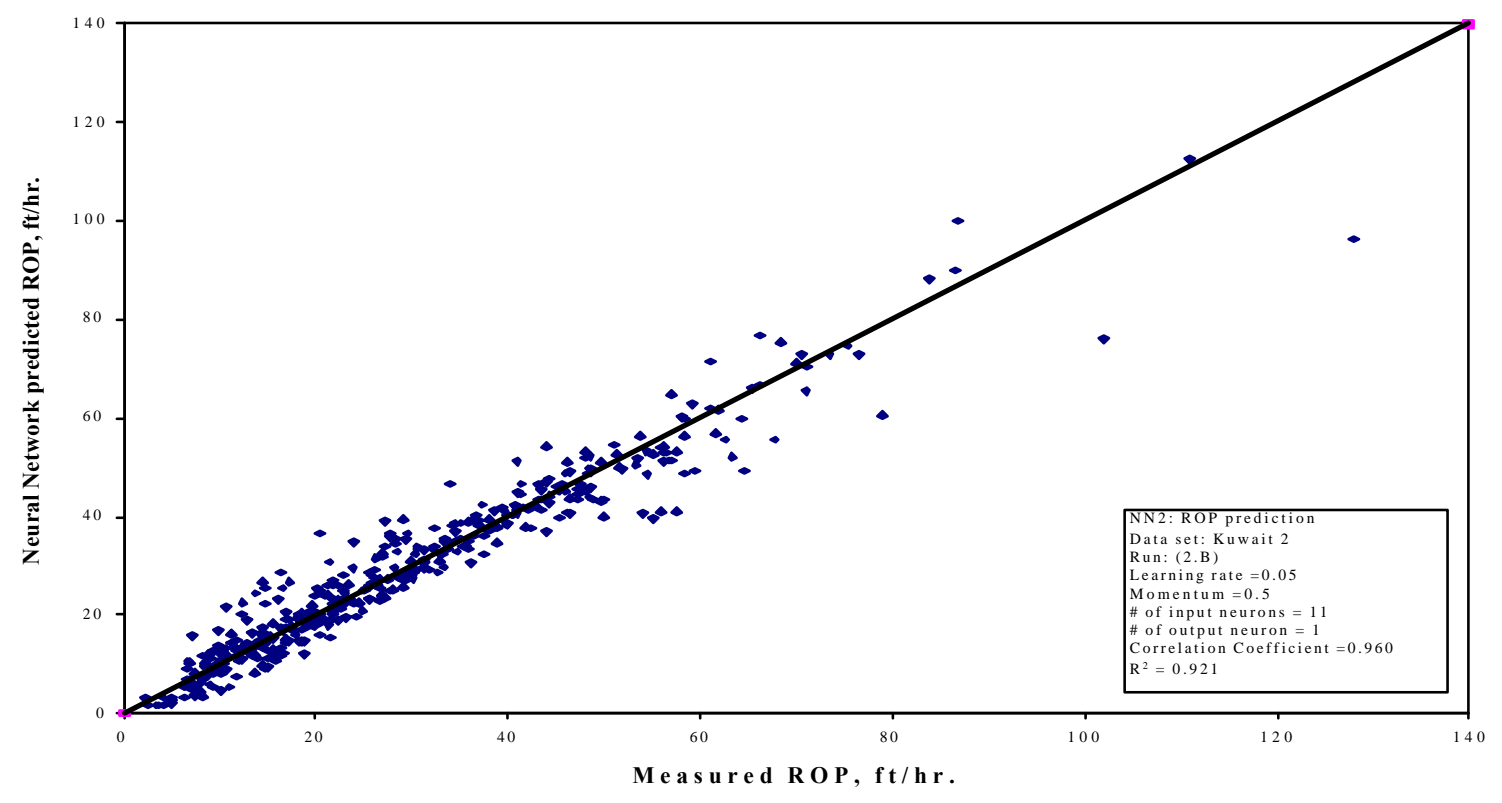

Figure 7. Comparison of neural network predicted ROP with the measured ROP. Kuwait-2 NN2 Run 2.B.

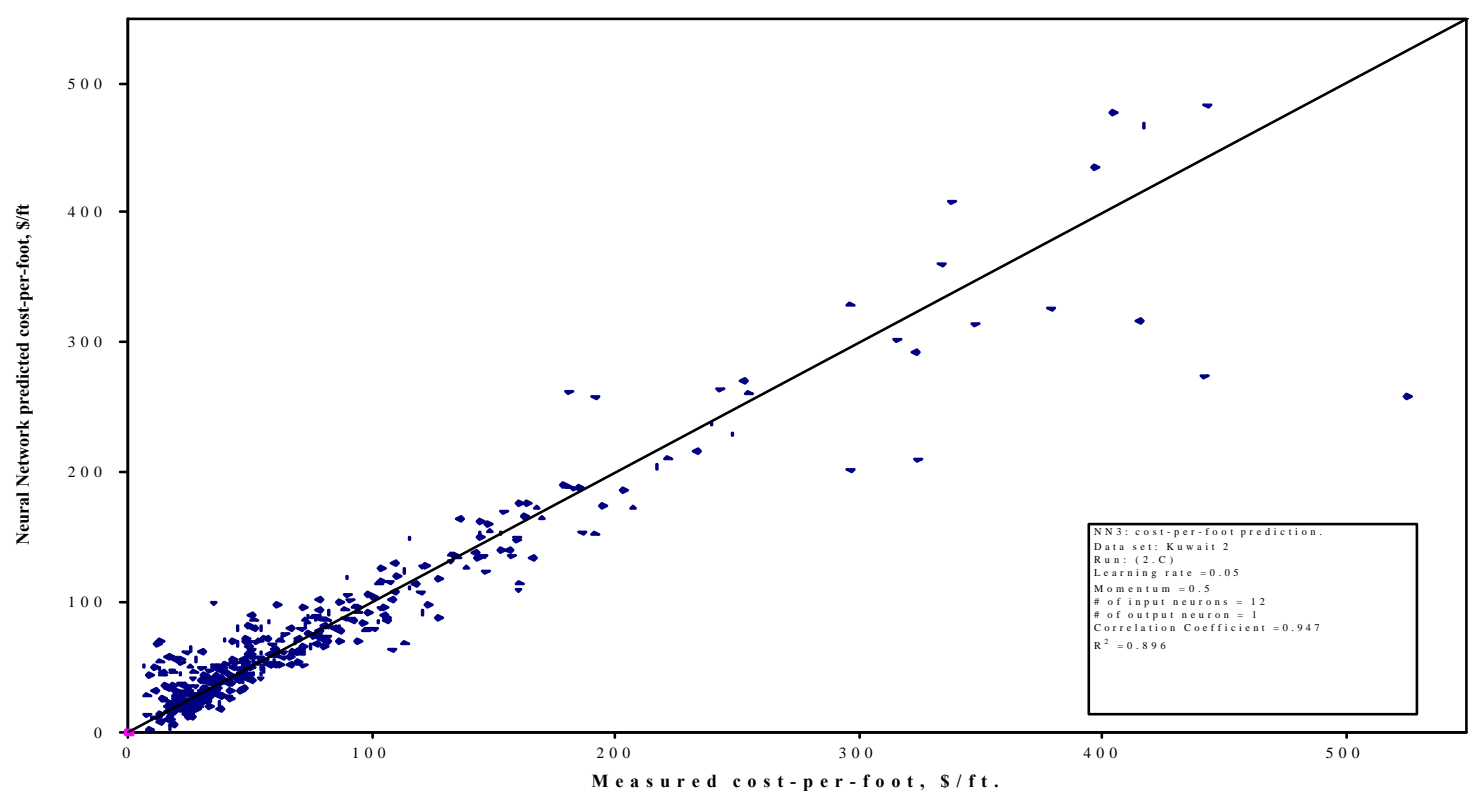

Figure 8. Comparison of neural netwo rk predicted cost-per-foot with the measured cost per foot. Kuwait-2 NN3 Run 2.C. 


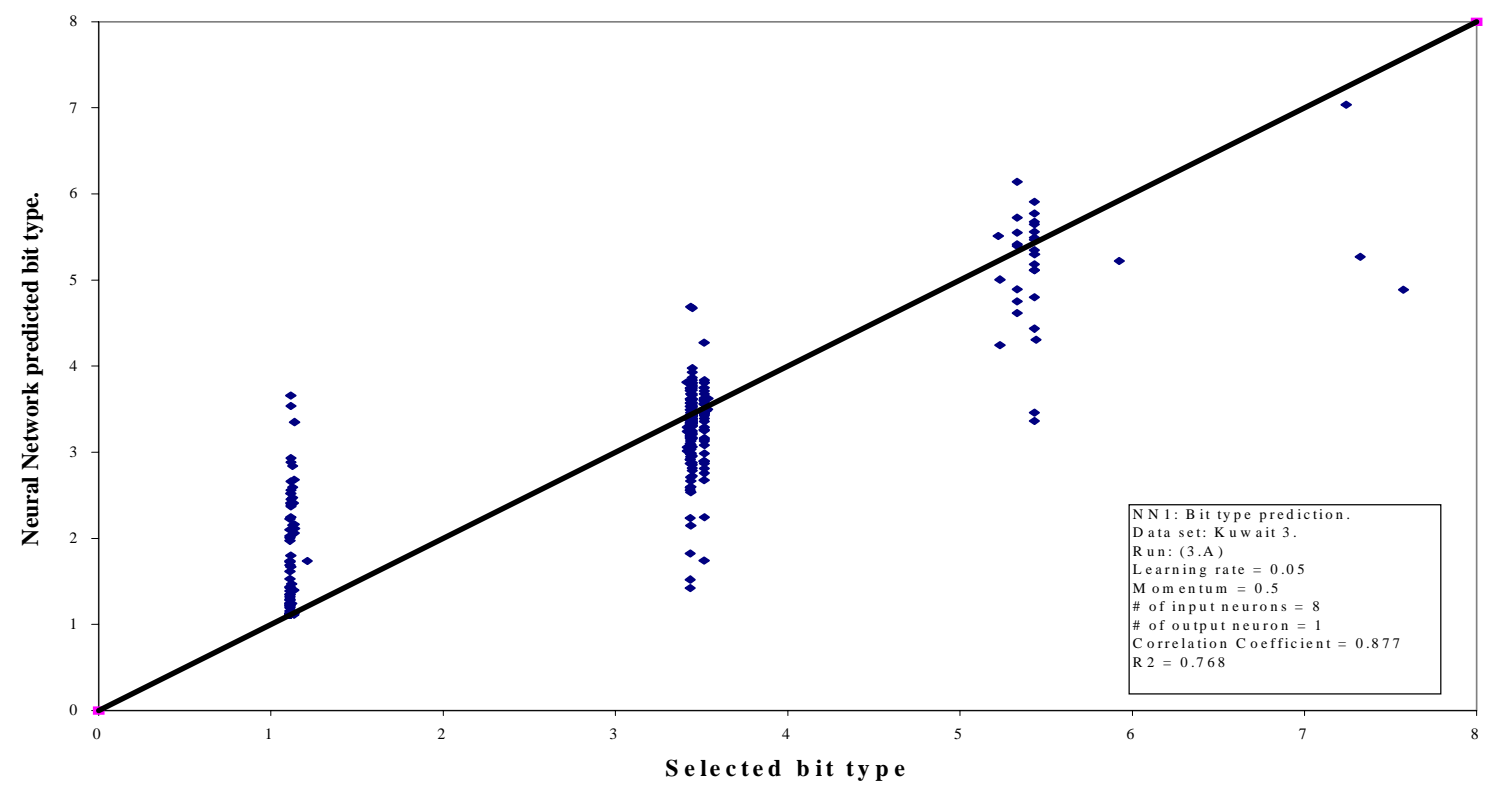

Figure 9. Comparison of neural network predicted bit type with the selected bit type. Kuwait-3 NN1 Run (3.A).

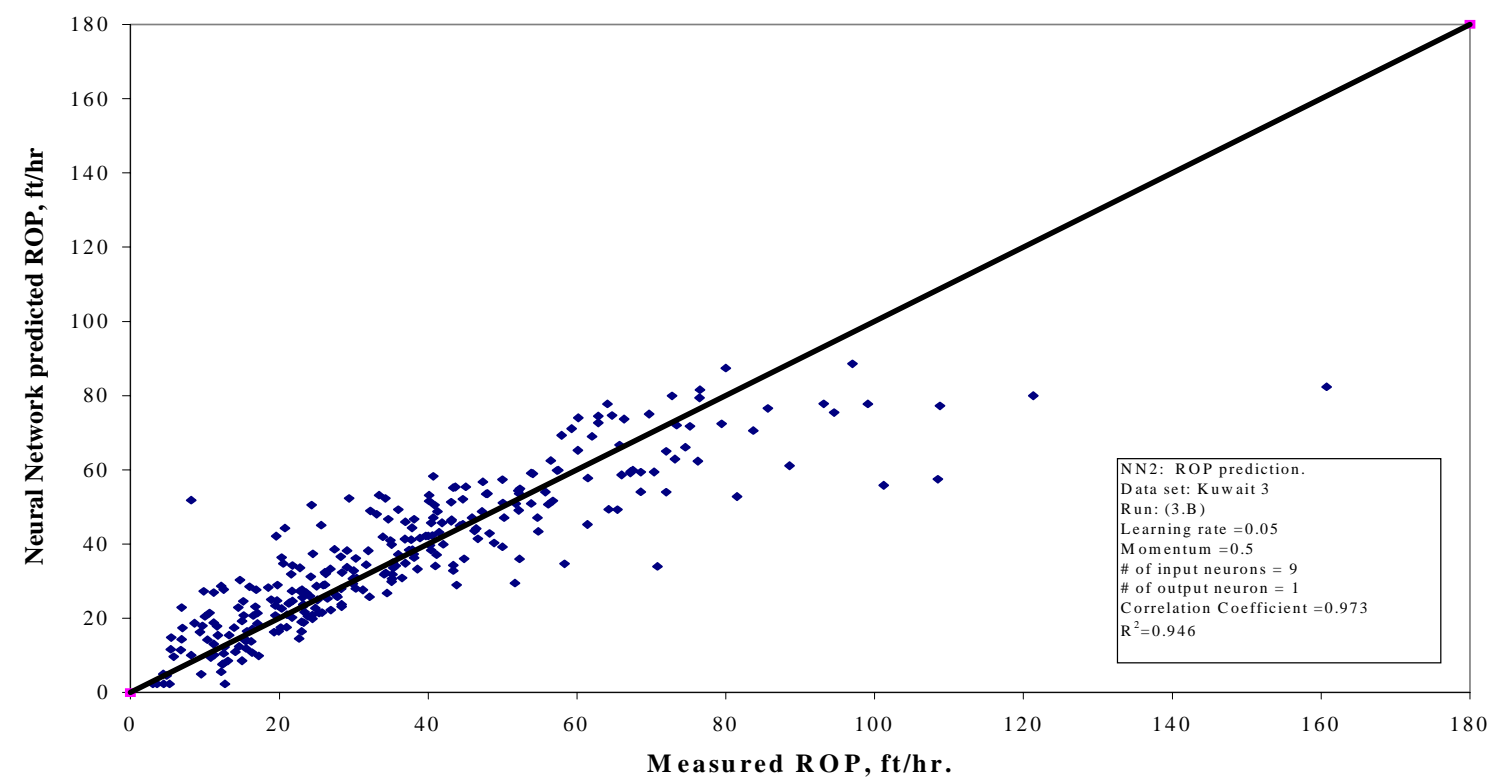

Figure 10. Comparison of neural network predicted ROP with the measured ROP. Kuwait-3 NN2 Run 3.B. 


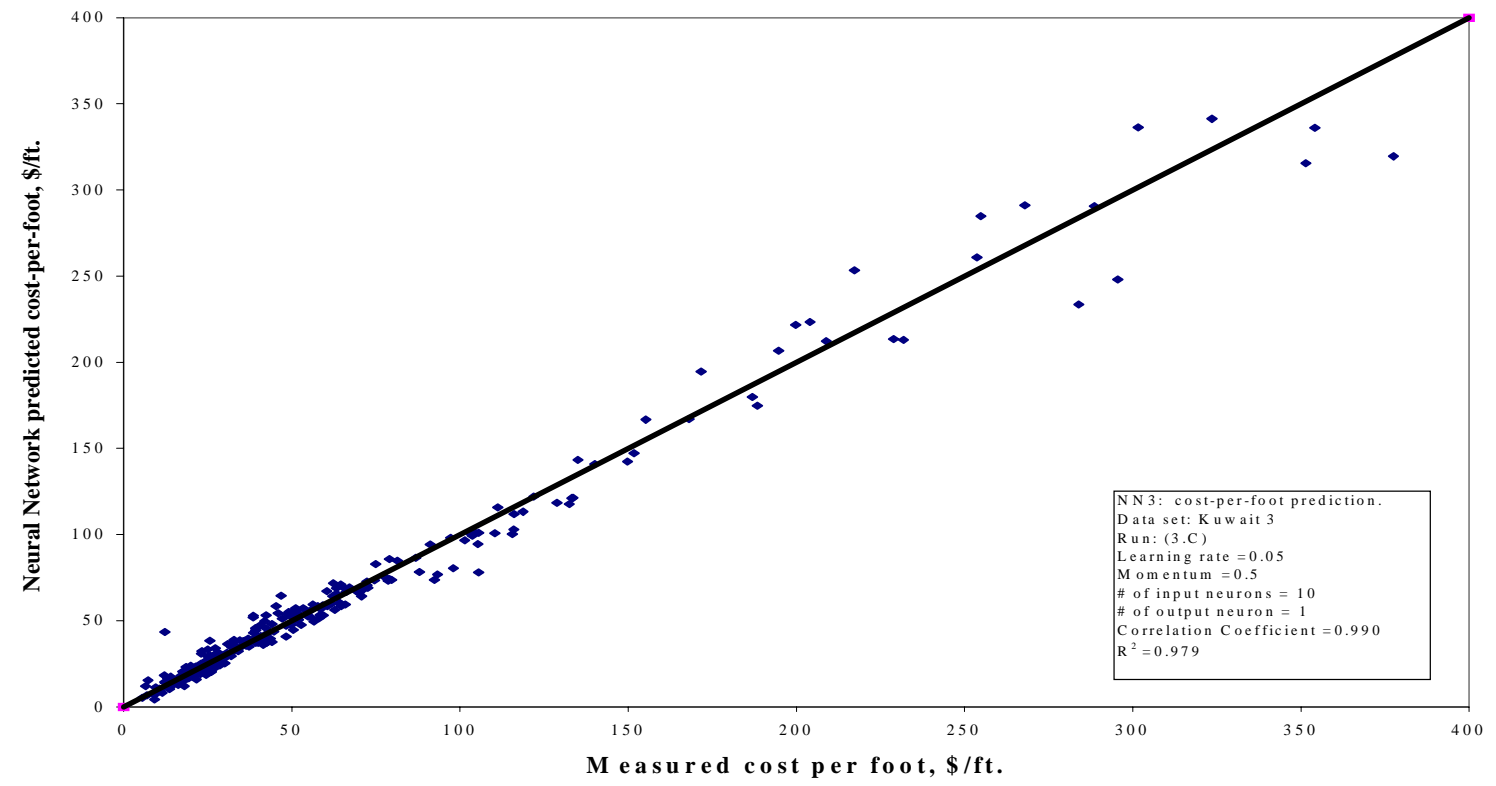

Figure 11. Comparison of neural network predicted cost per foot with the measured cost per foot. Kuwait-3 NN3 Run 3.C.

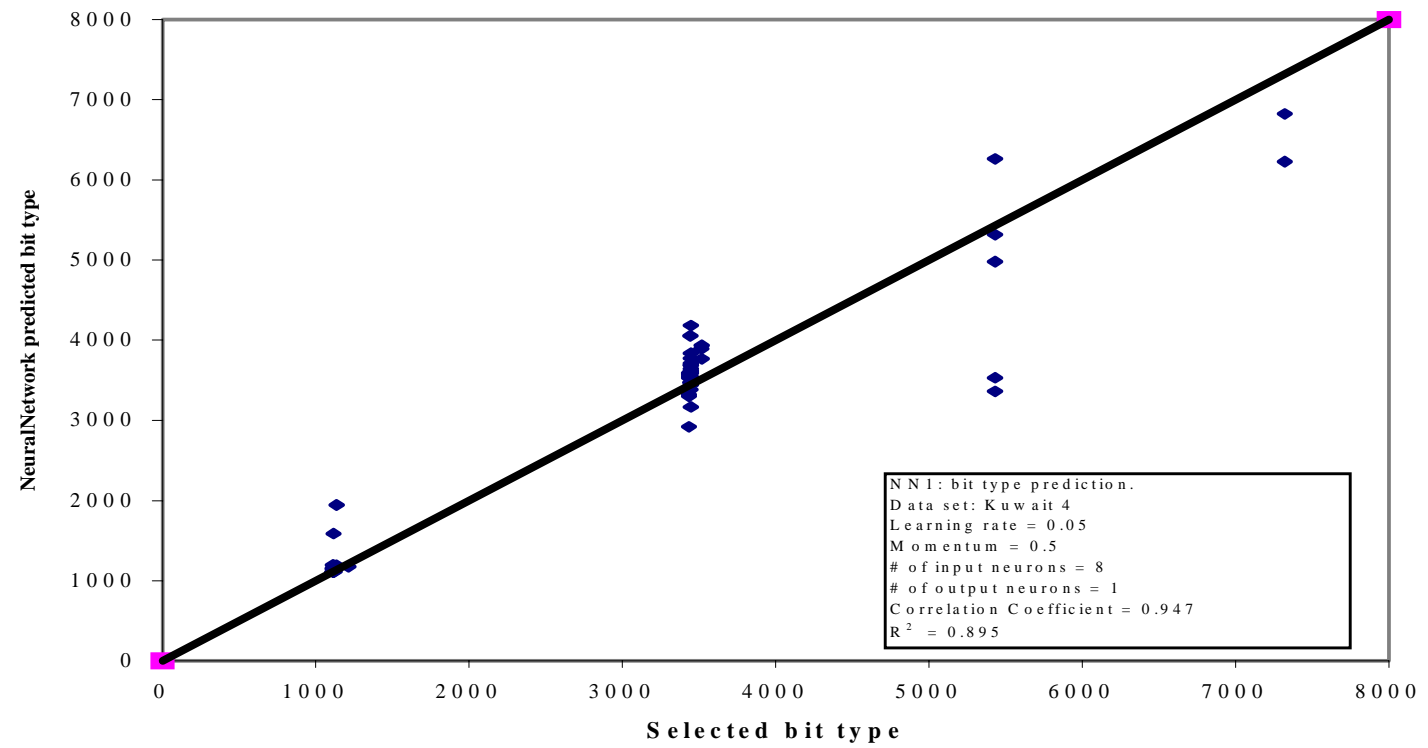

Figure 12. Comparison of neural network predicted bit type with the selected bit type. Using bit type code number 6, (V2 code). Kuwait-4 NN1 Run 4. 


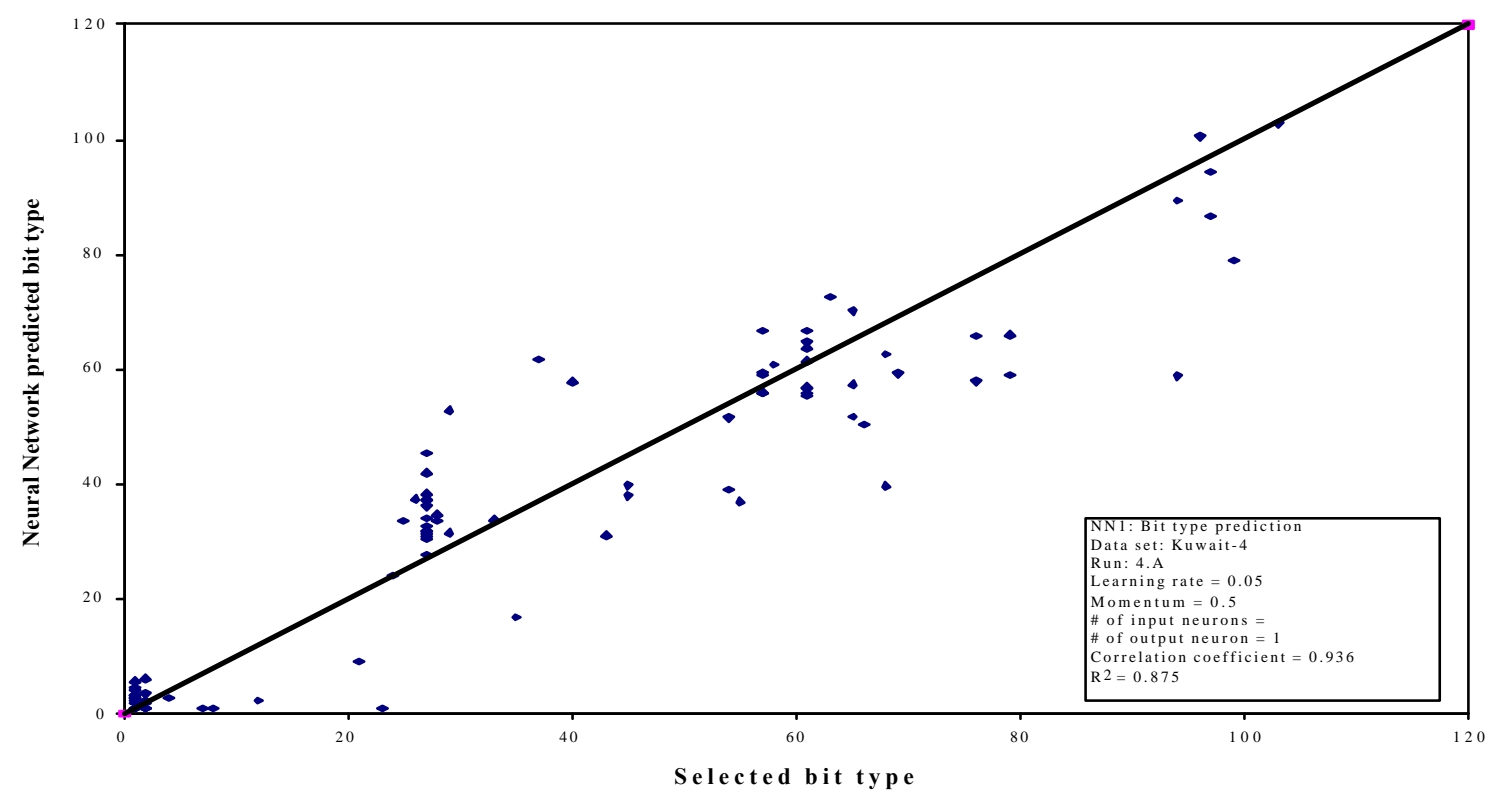

Figure 13. Comparison of neural network predicted bit type with the selected bit type. Using bit type code number 9, (bit code). Kuwait-4 NN1 Run 4.A.

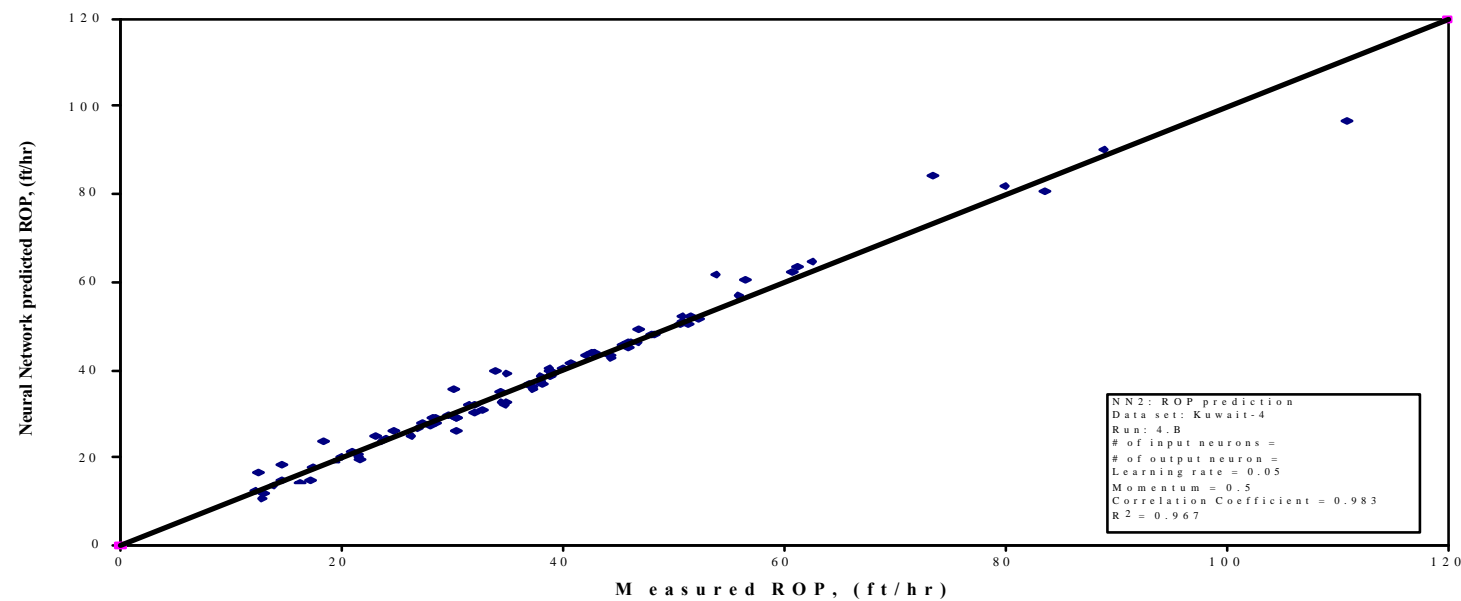

Figure 14. Comparison of neural network predicted ROP with the measured ROP. Kuwait-4 NN2 Run 4.B. 


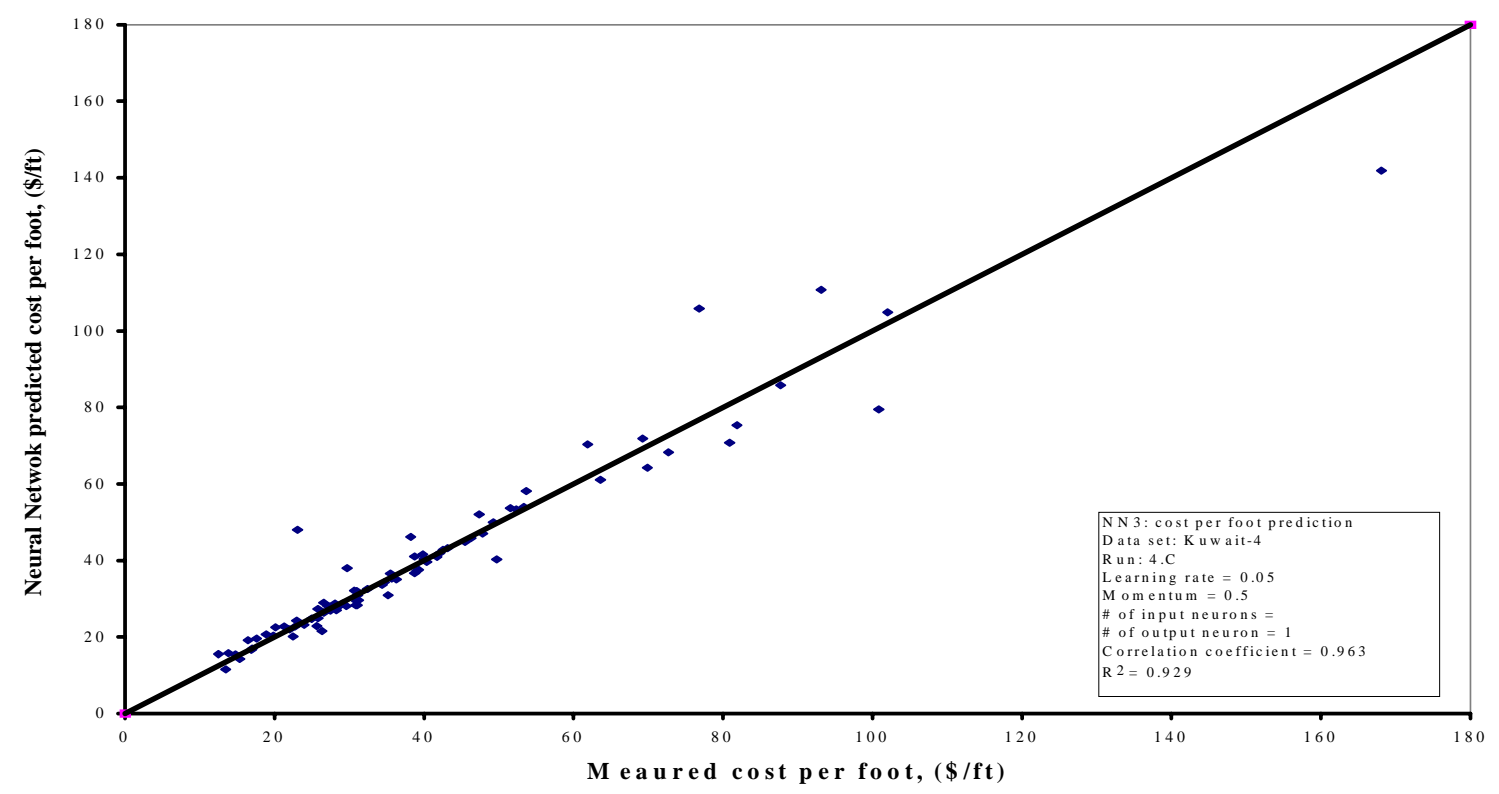

Figure 15. Comparison of neural network predicted cost per foot with the measured cost per foot. Kuwait-4 NN3 Run 4.C.

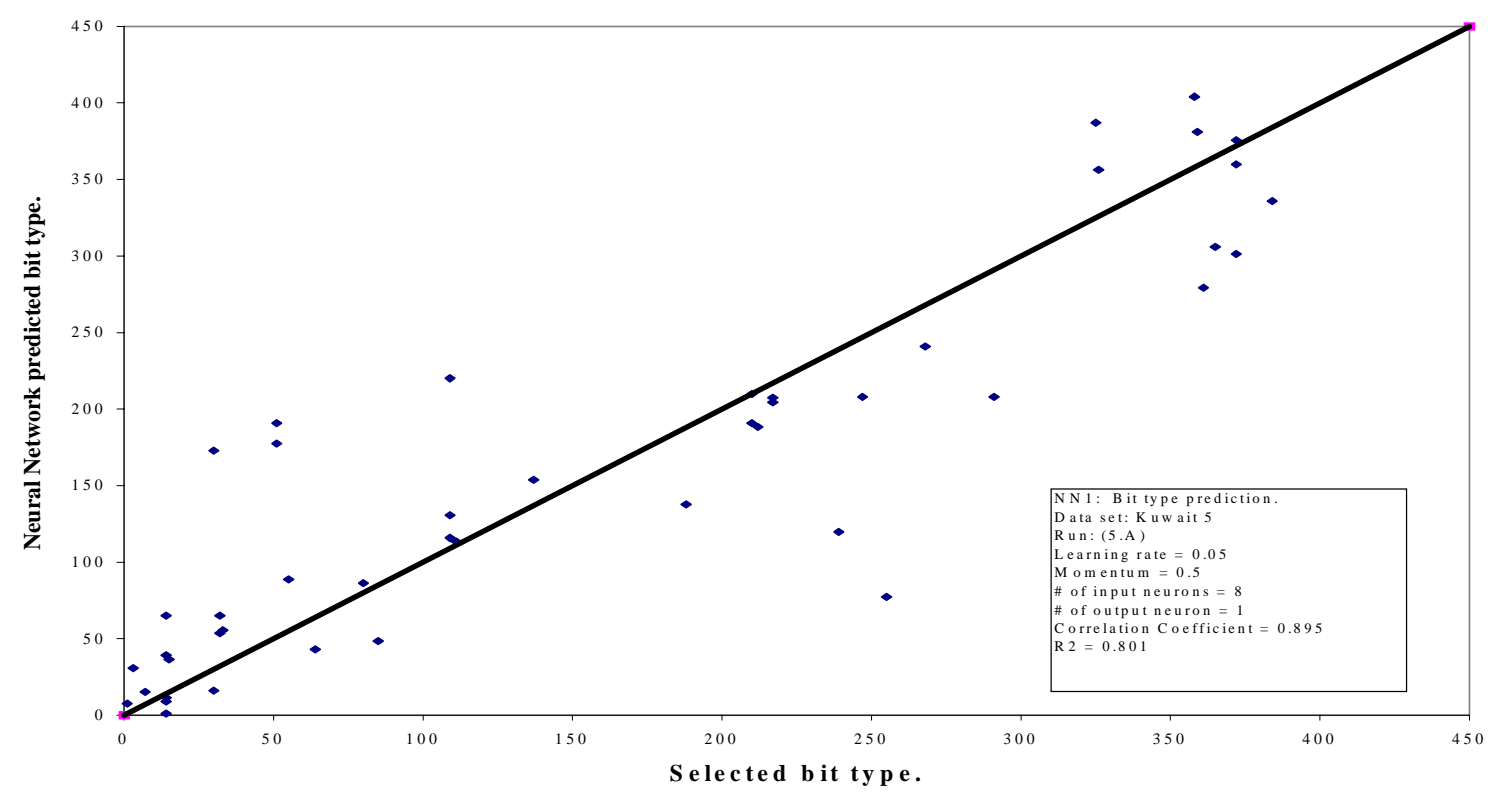

Figure 16. Comparison of neural network predicted bit type with the selected bit type. Kuwait-5 NN1 Run 5.A. 


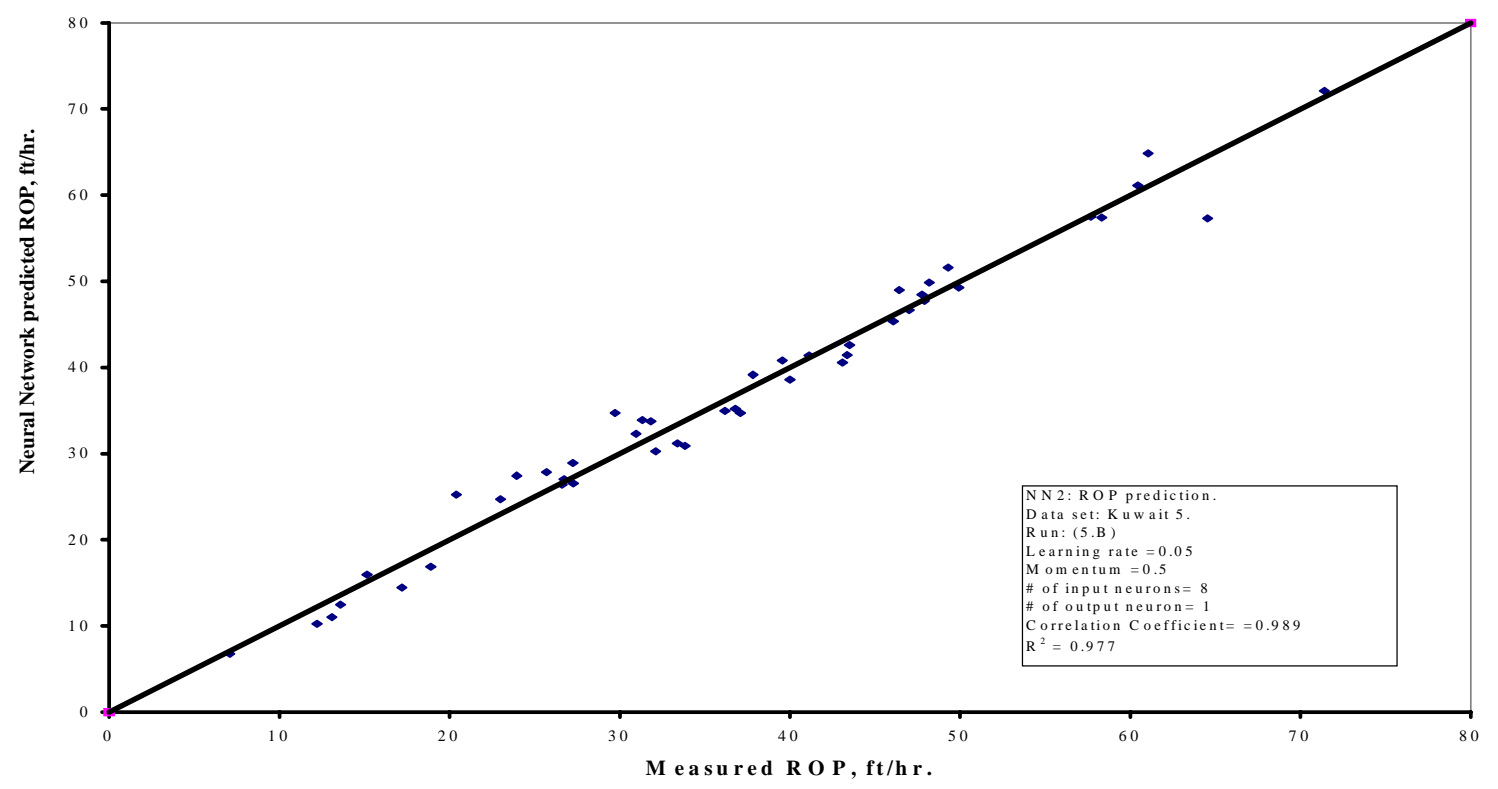

Figure 17. Comparison of neural network predicted ROP with the measured ROP. Kuwait-5 Run 5.B.

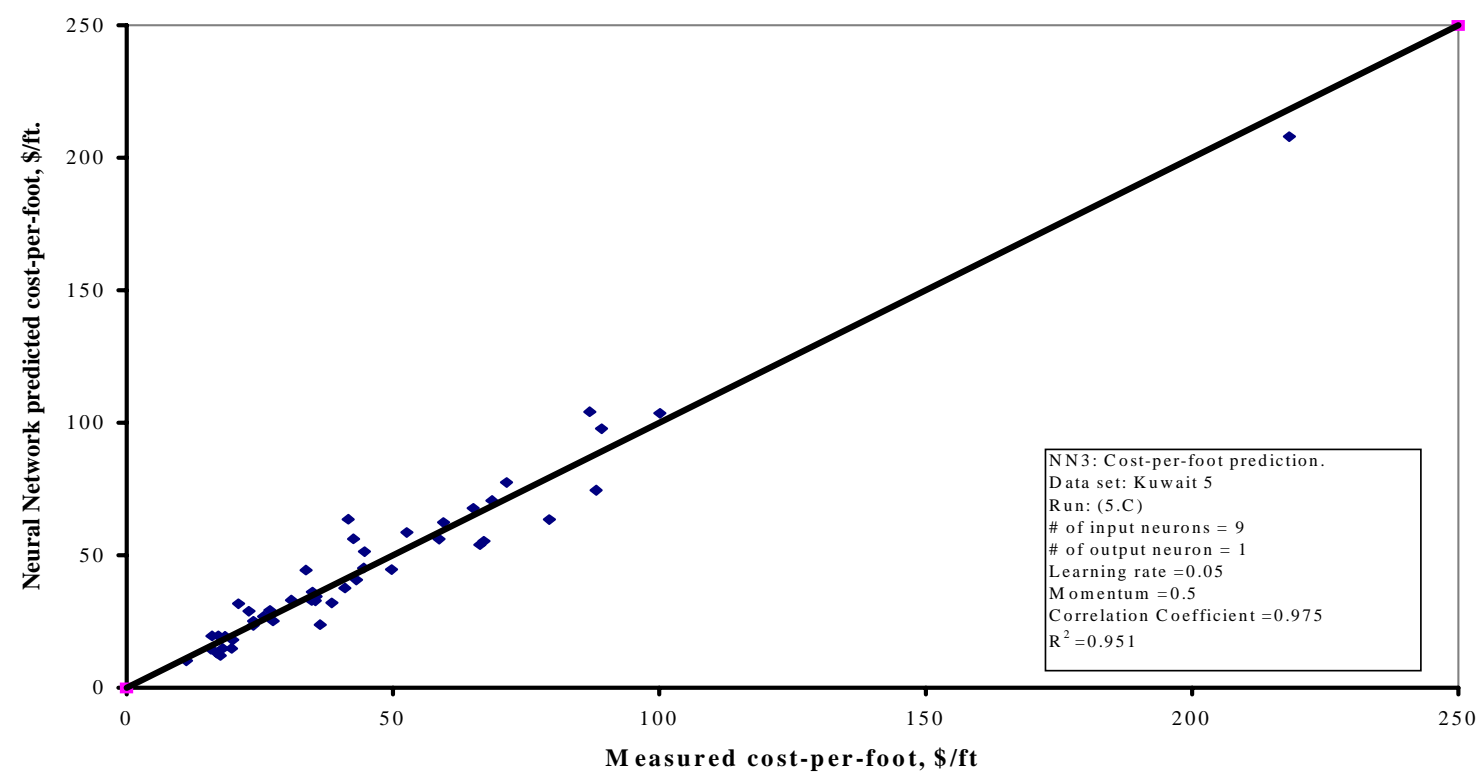

Figure 18. Comparison of neural network predicted cost per foot with the measured cost per foot. Kuwait-5 NN3 Run 5.C. 


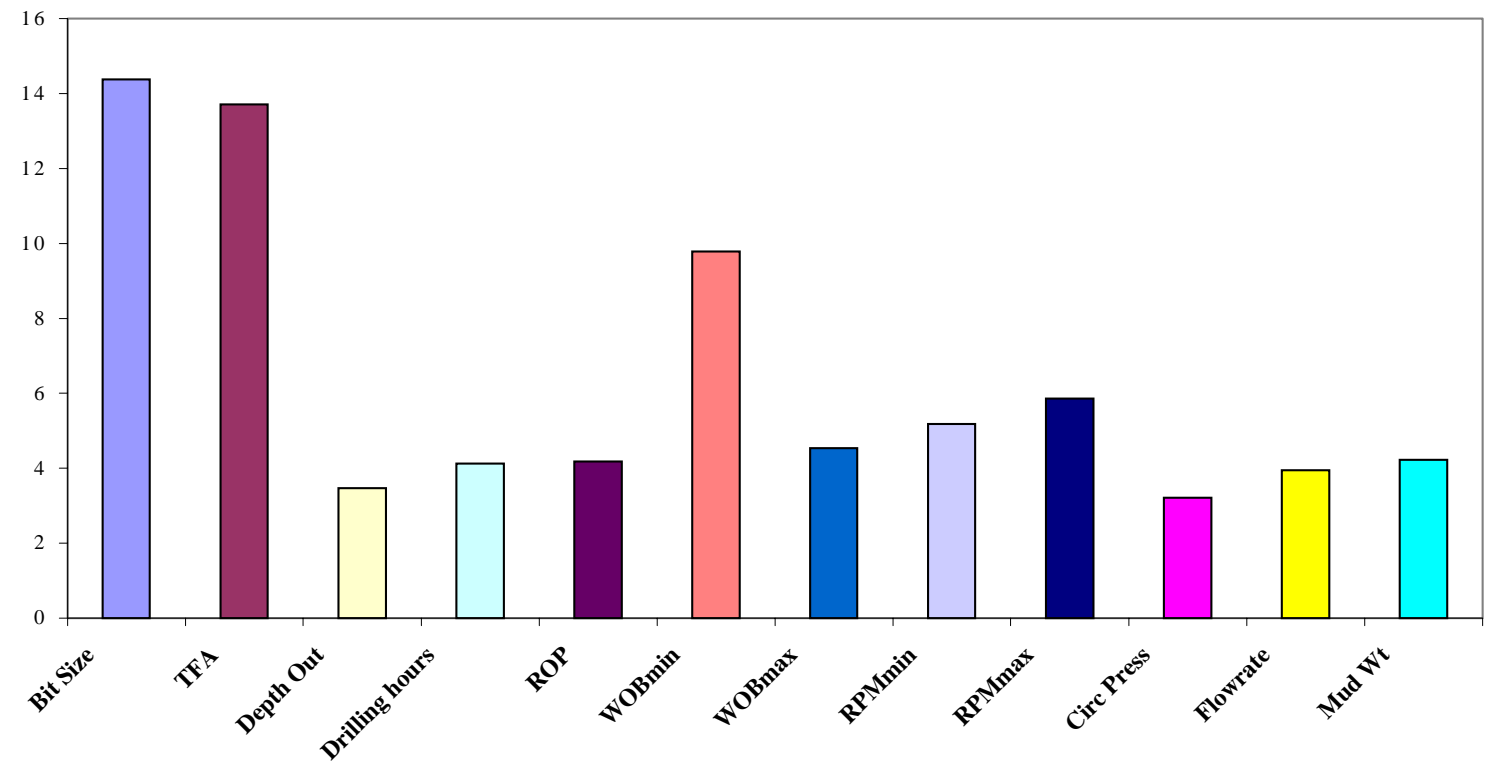

Figure 19. Contribution of input parameters for the bit type prediction. Kuwait-1 NN1 Run 1.A

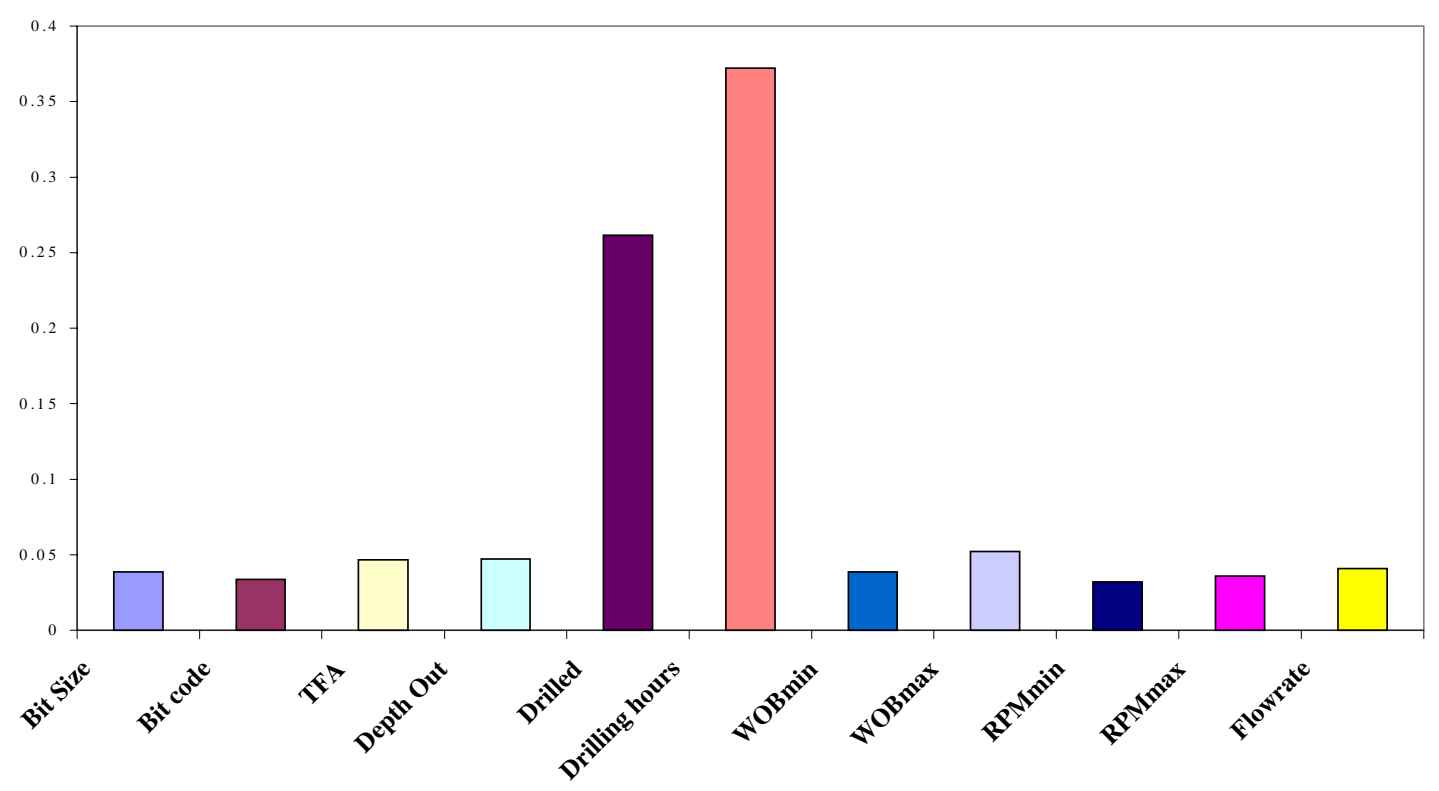

Figure 20. Contribution of input parameters for the ROP prediction. Kuwait-1 NN2 Run 1.B. 


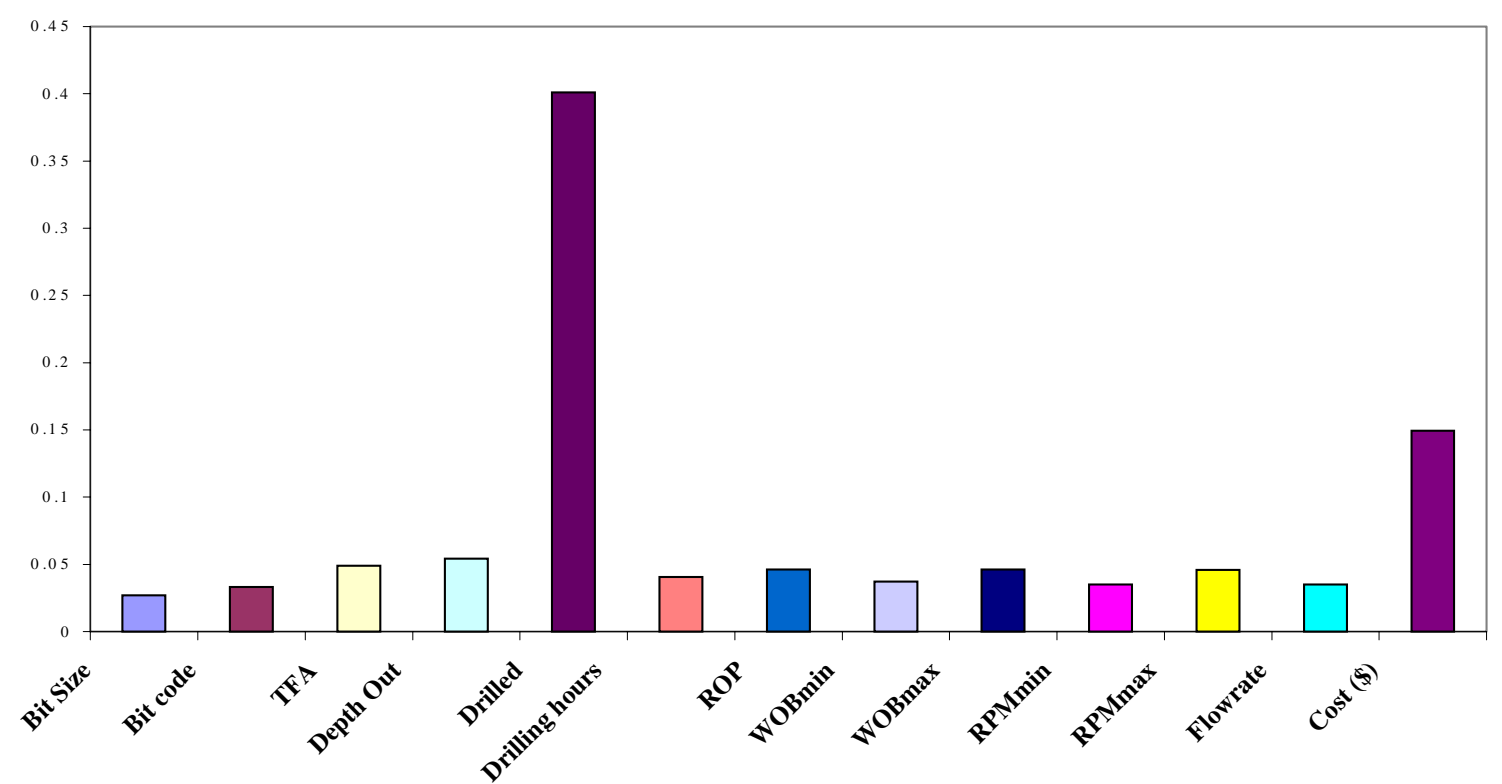

Figure 21. Contribution of input parameters for the cost per foot prediction. Kuwait-1 NN3 Run 1.C.

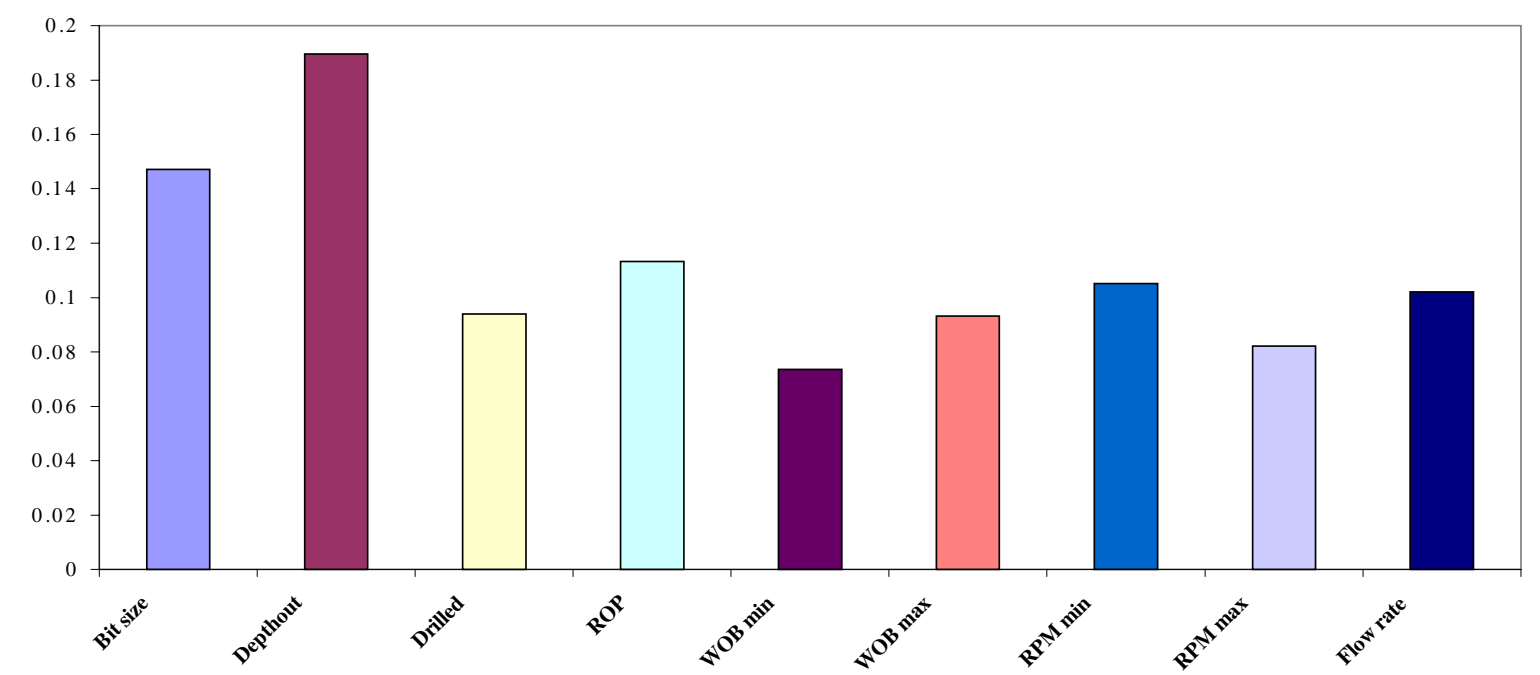

Figure 22. Contribution of input parameters for the bit type prediction. Kuwait-2 NN1 Run 2.A. 


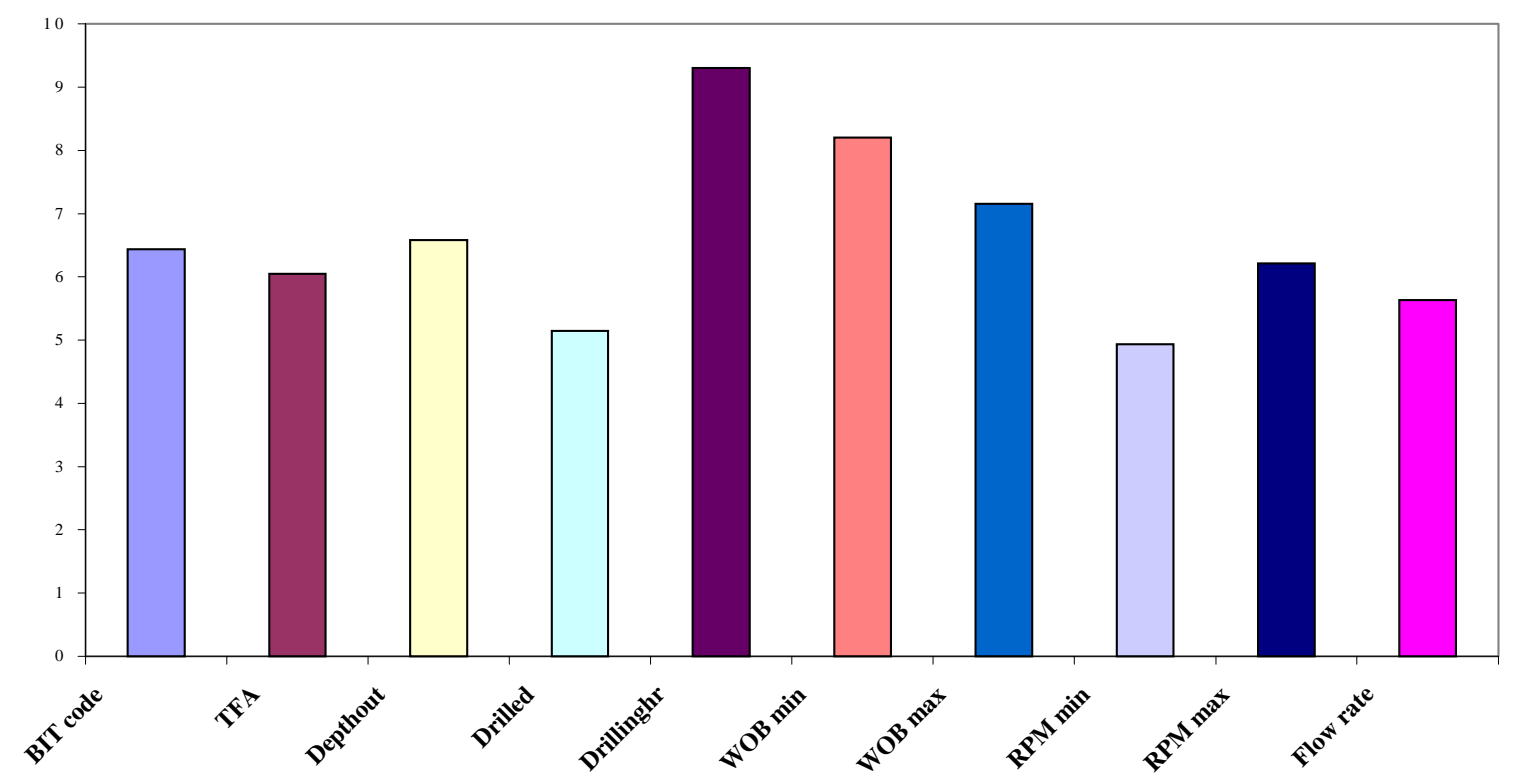

Figure 23. Contribution of input parameters for the ROP prediction. Kuwait-2 NN2 Run 2.B.

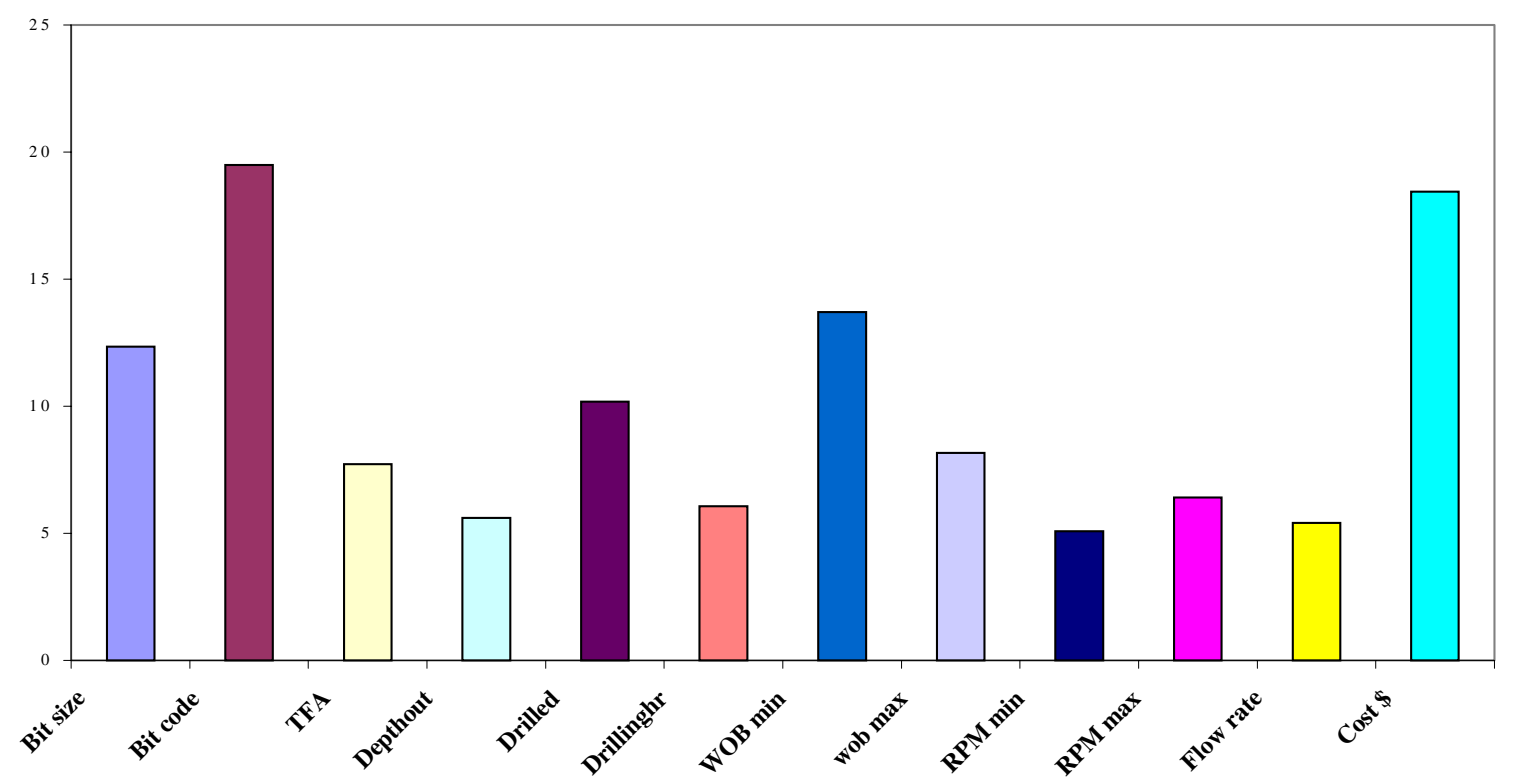

Figure 24. Contribution of input parameters for the cost per foot prediction. Kuwait-2 NN3 Run 2.C. 


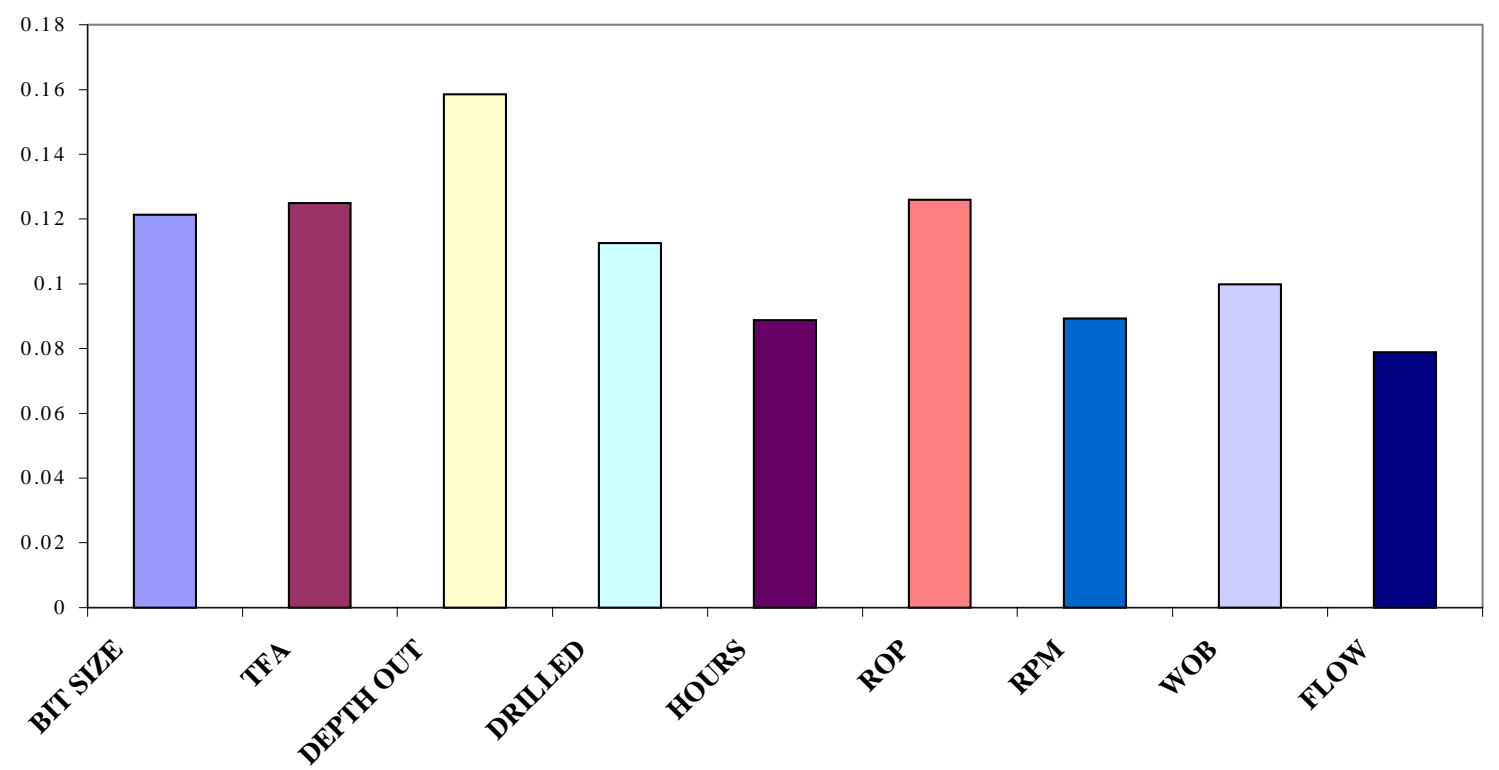

Figure 25. Contribution of input parameters for the bit type prediction. Kuwait-3 NN1 Run 3.A.

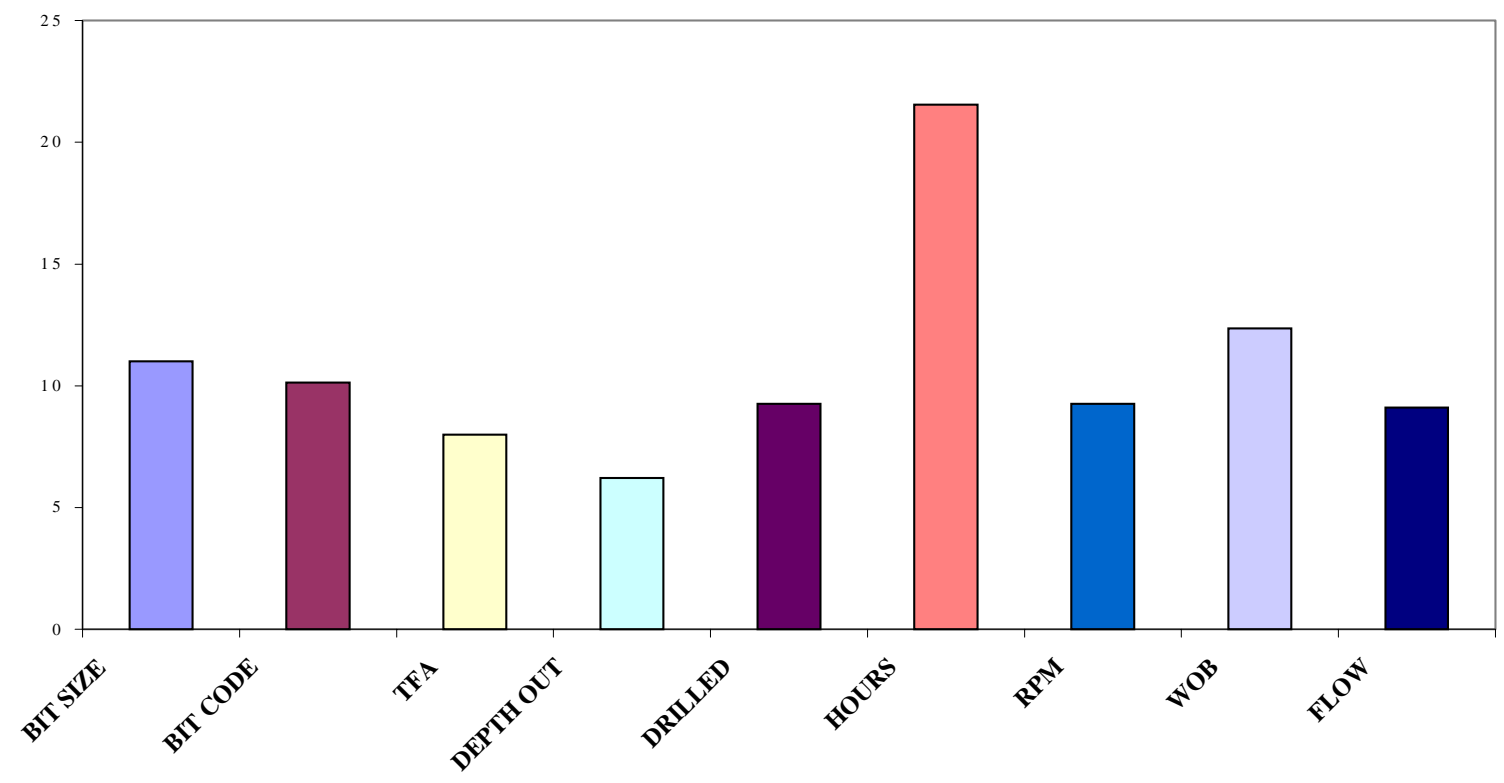

Figure 26. Contribution of input parameters for the ROP prediction.

Kuwait-3 NN2 Run 3.B. 


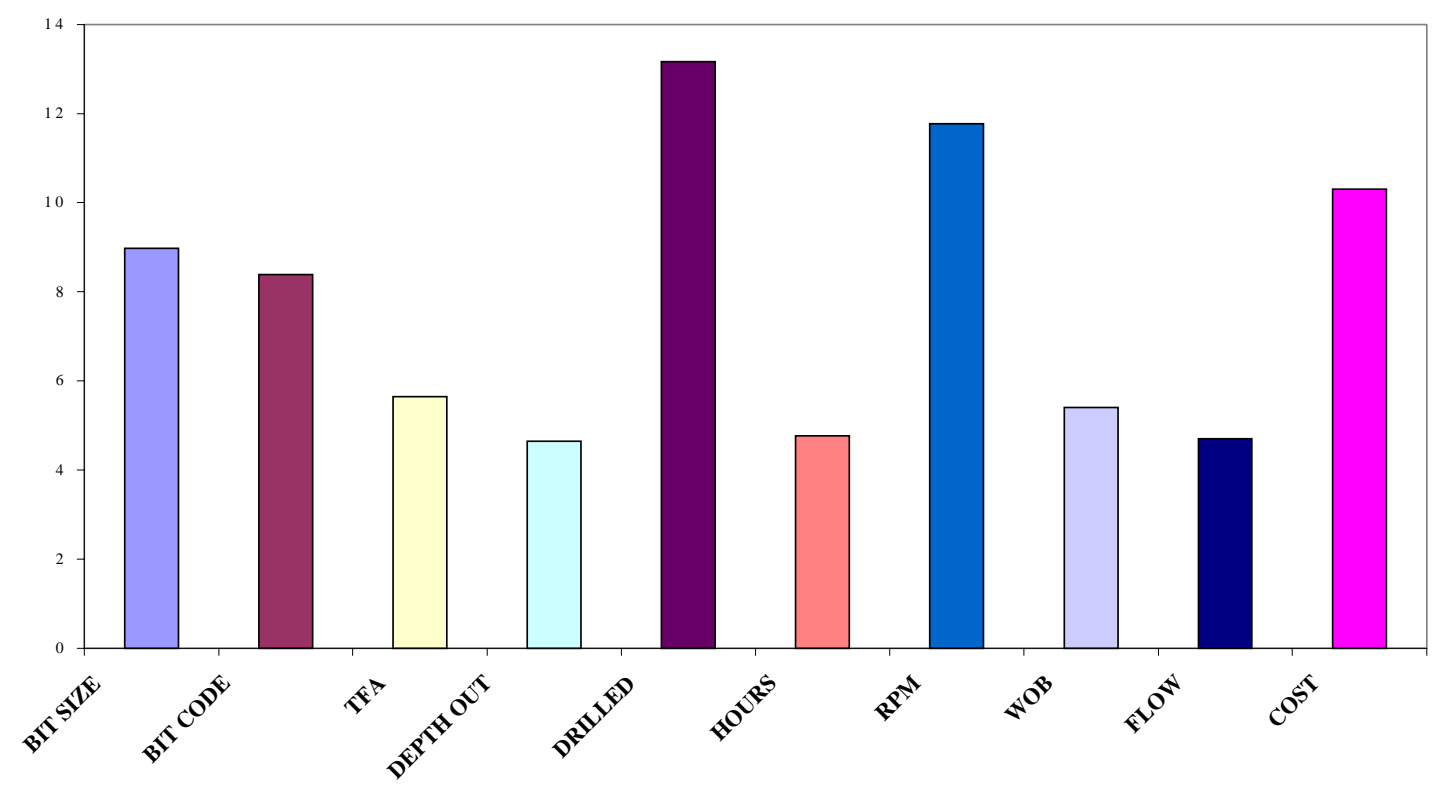

Figure 27. Contribution of input parameters for the cost per foot prediction. Kuwait-3 NN3 Run 3.C.

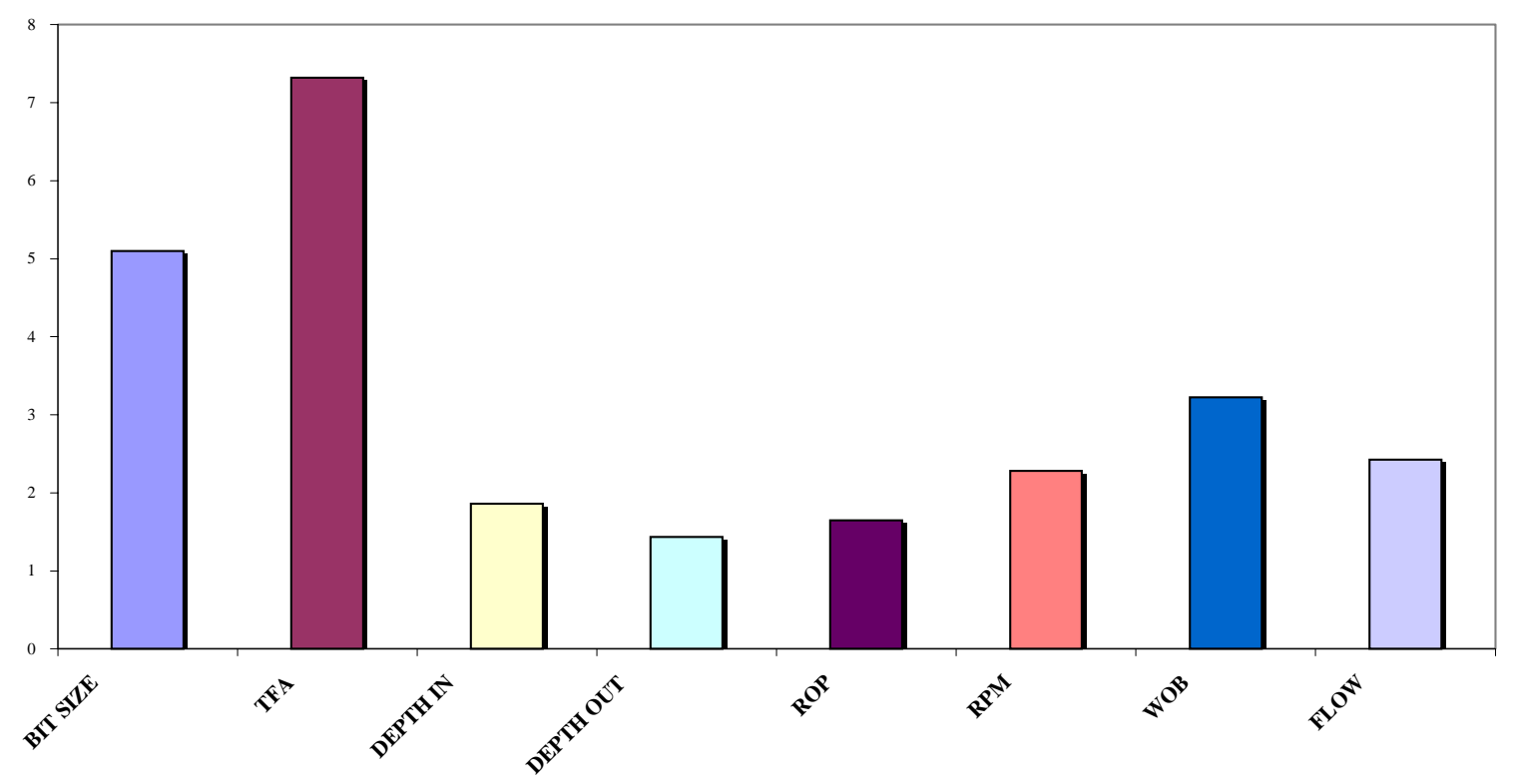

Figure 28. Contribution of input parameters for the bit type prediction. Using bit type code number 6, V2 code. Kuwait-4 NN1 Run 4. 


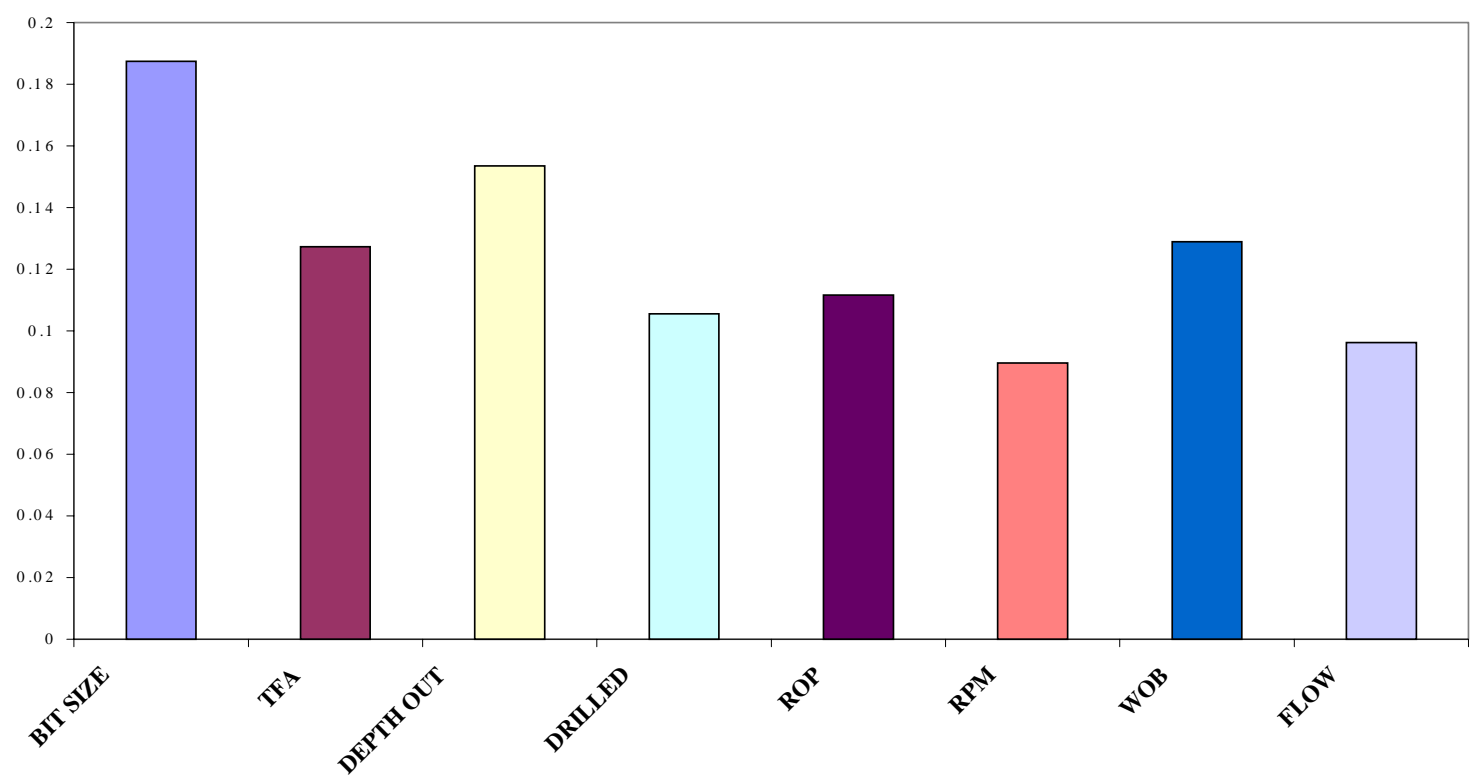

Figure 29. Contribution of input parameters for the bit type prediction. Using bit type code number 9, bit code. Kuwait-4 NN1 Run 4.A.

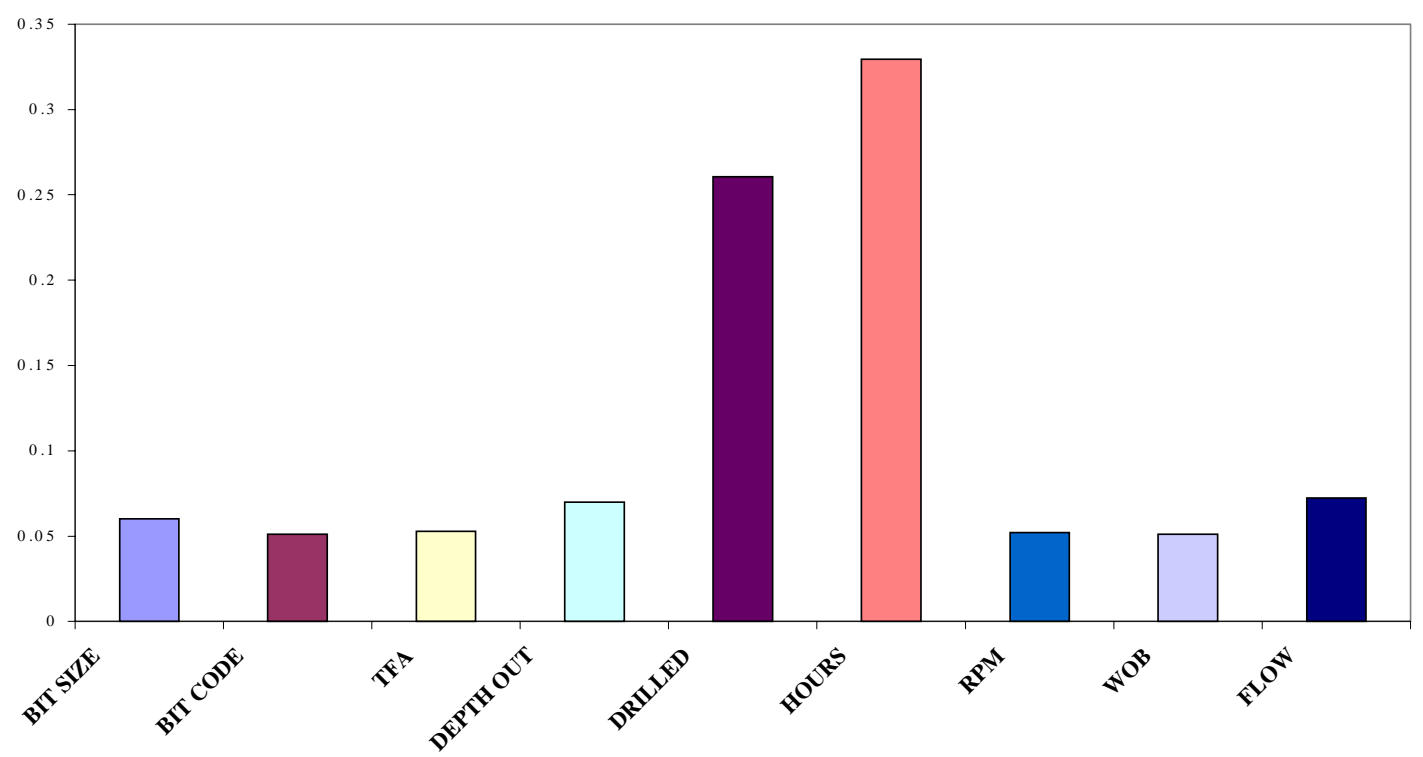

Figure 30. Contribution of input parameters for the ROP prediction.

Kuwait-4 NN2 Run 4.B. 


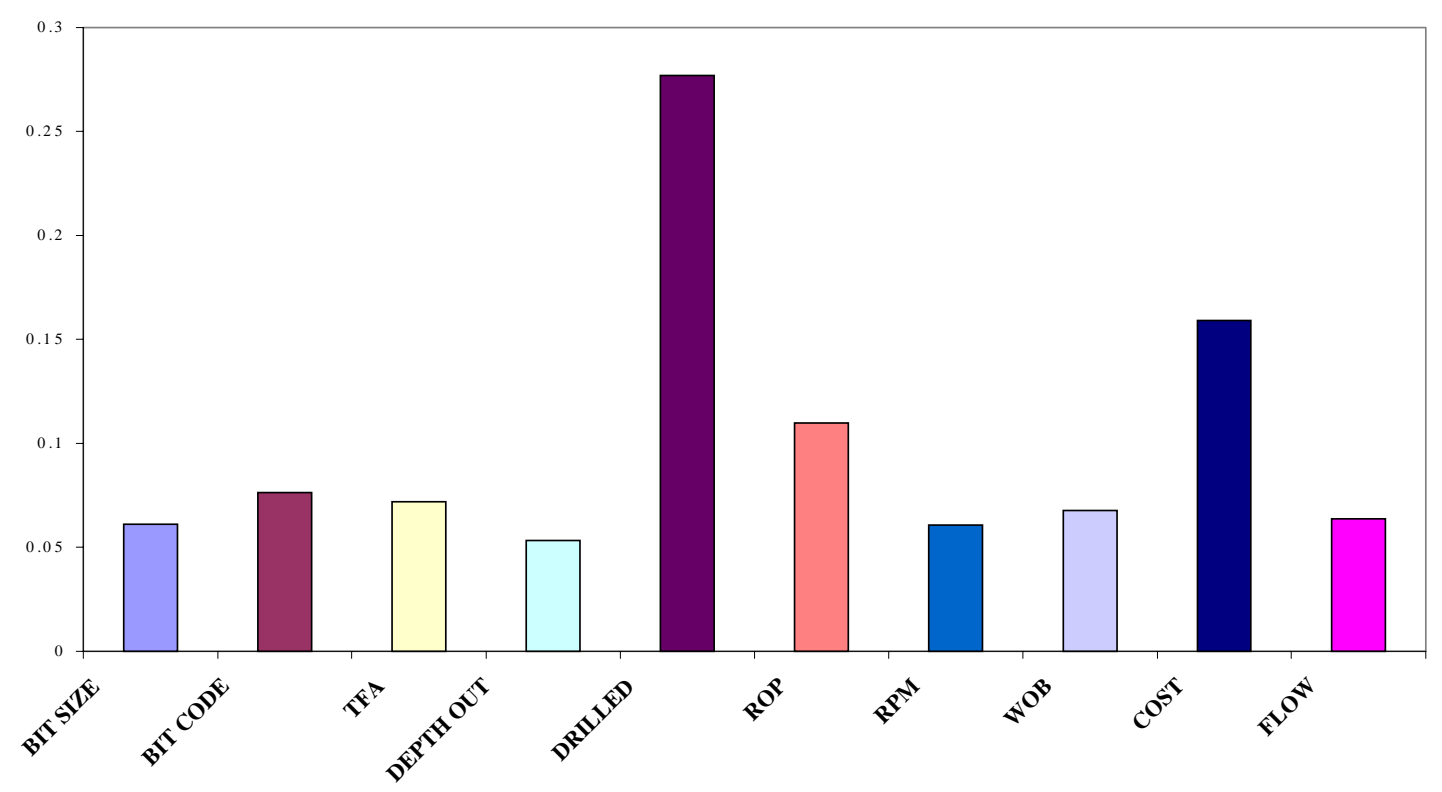

Figure 31. Contribution of input parameters for the cost per foot prediction. Kuwait-4 NN3 Run 4.C.

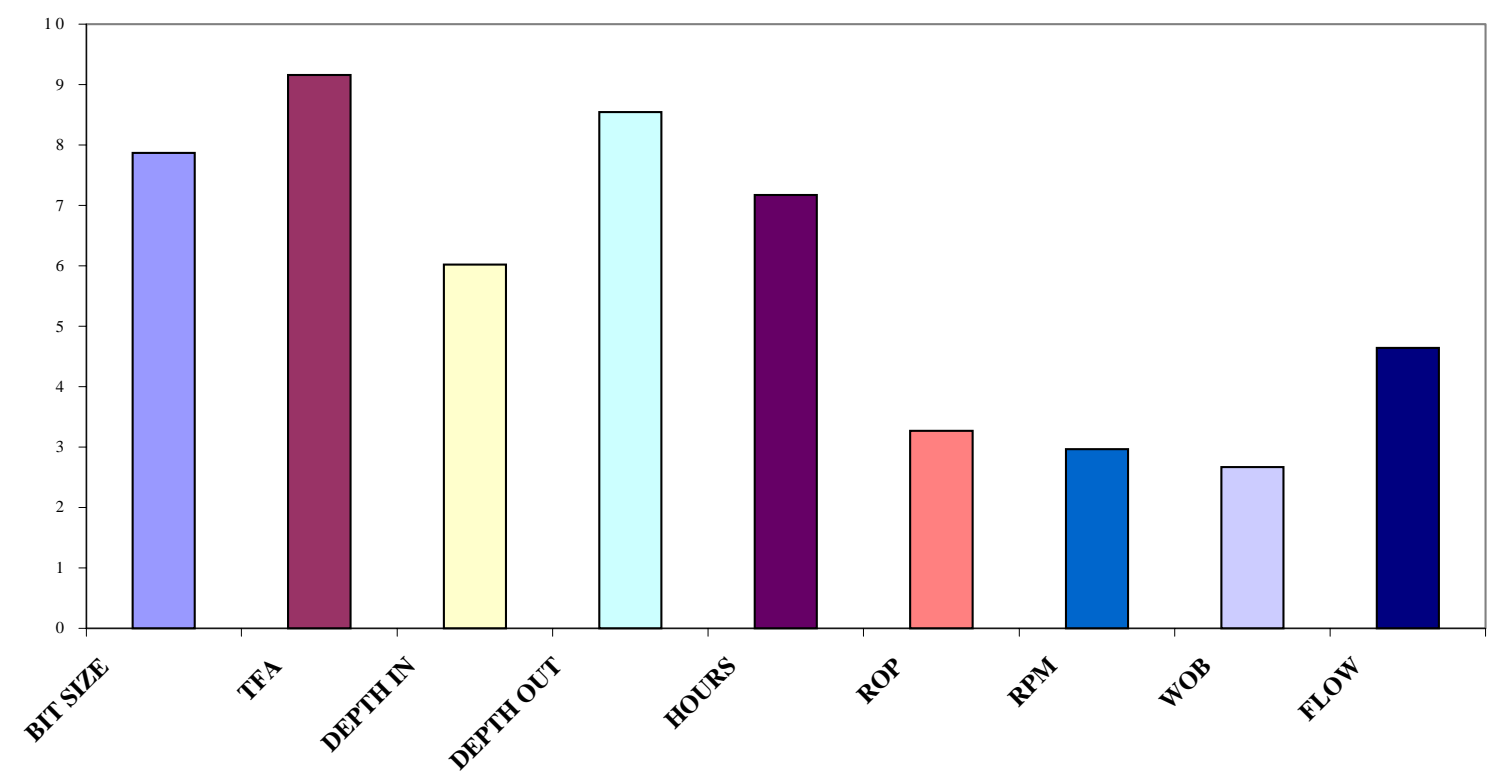

Figure 32. Contribution of input parameters for the bit type prediction. Kuwait-5 NN1 Run 5.A. 


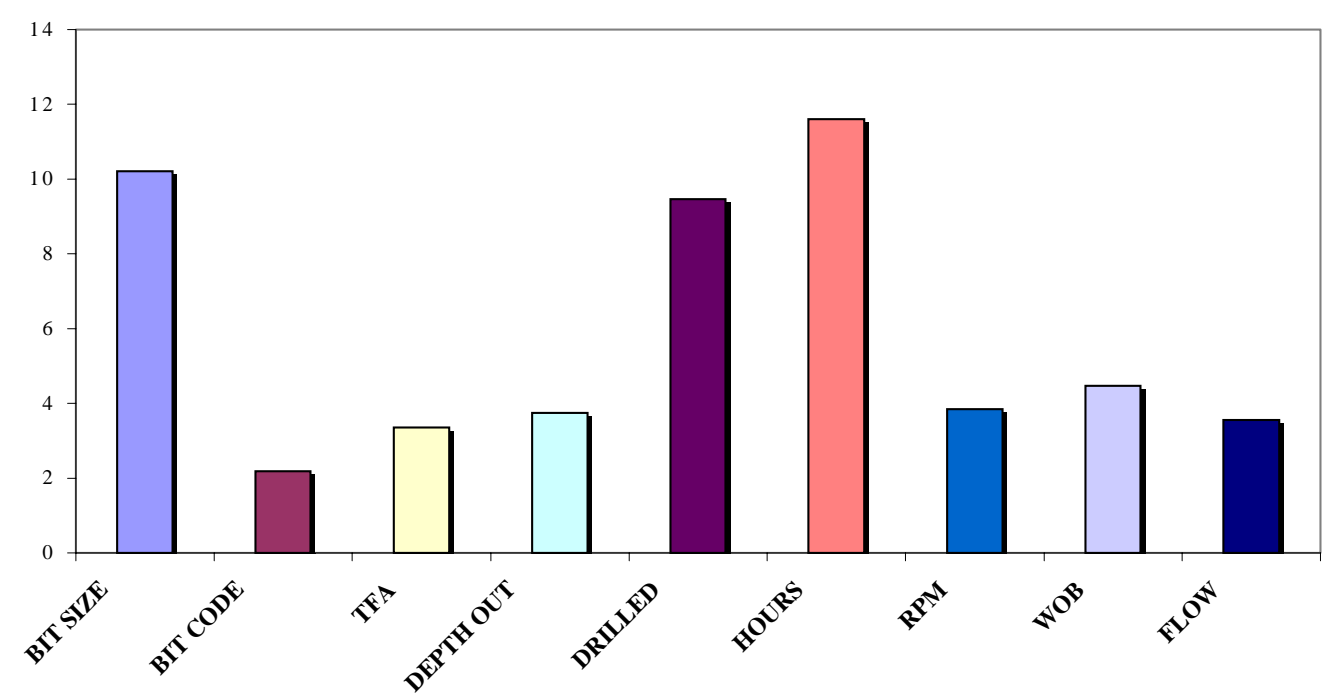

Figure 33. Contribution of input parameters for the ROP prediction. Kuwait-5 NN2 Run 5.B.

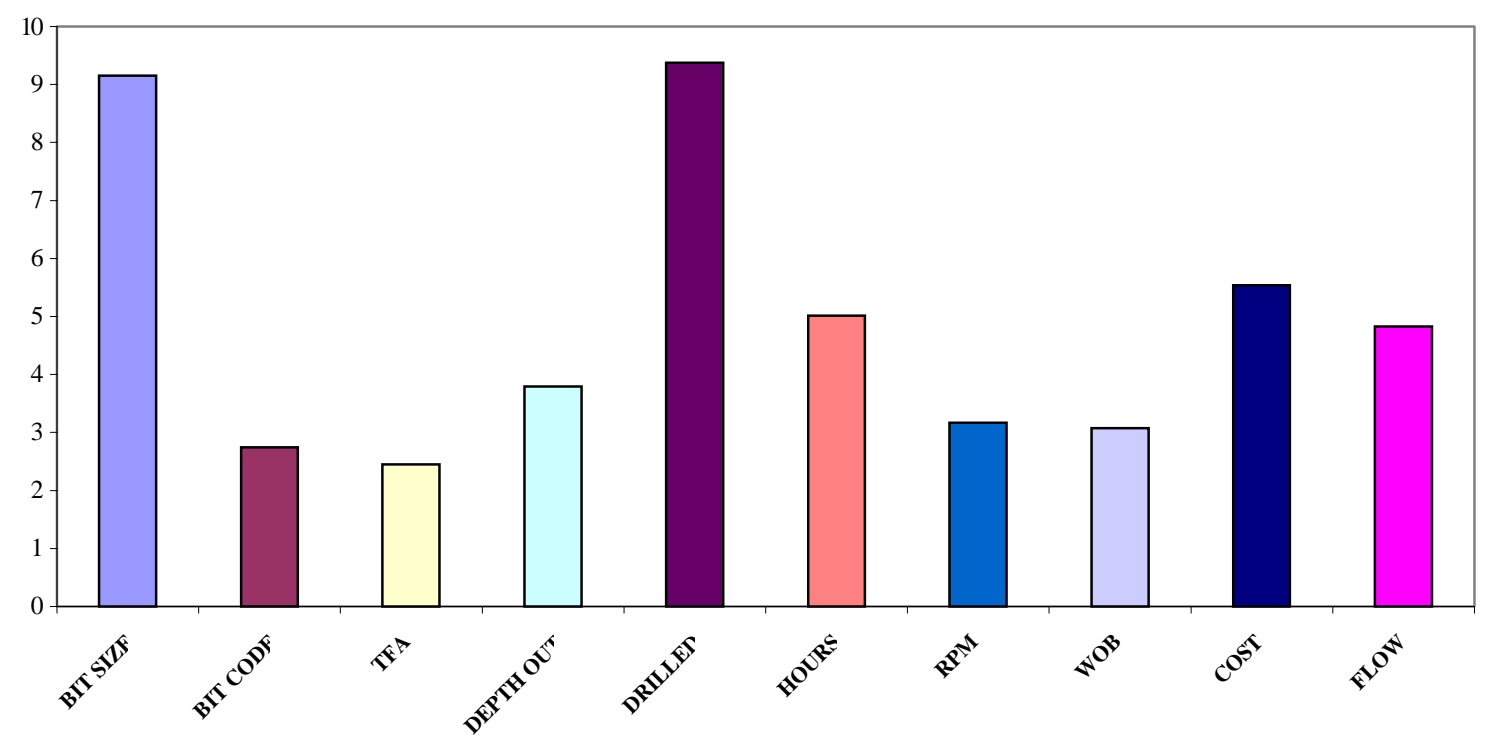

Figure 34. Contribution of input parameters for the cost per foot prediction. Kuwait-5 NN3 Run 5.C. 